\title{
SUBVARIETIES OF MODULI SPACE DETERMINED BY FINITE GROUPS ACTING ON SURFACES
}

\author{
JOHN F. X. RIES
}

\begin{abstract}
Suppose the finite group $G$ acts as orientation preserving homeomorphisms of the oriented surface $S$ of genus $g$. This determines an irreducible subvariety $\mathscr{K}_{g}^{[G]}$ of the moduli space $\mathscr{M}_{g}$ of Riemann surfaces of genus $g$ consisting of all surfaces with a group $G_{1}$ of holomorphic homeomorphisms of the same topological type as $G$. This family is determined by an equivalence class of epimorphisms $\psi$ from a Fuchsian group $\Gamma$ to $G$ whose kernel is isomorphic to the fundamental group of $S$. To examine the singularity of $\mathscr{M} g$ along this family one needs to know the full automorphism group of a generic element of $\mathscr{K}_{g}^{[G]}$. In $\S 2$ we show how to compute this from $\psi$. Let $\mathscr{M}_{g}^{G}$ denote the locus of all Riemann surfaces of genus $g$ whose automorphism group contains a subgroup isomorphic to $G$. In $\S 3$ we show that the irreducible components of this subvariety do not necessarily correspond to the families above, that is, the components cannot be put into a one-to-one correspondence with the topological actions of $G$. In $\S 4$ we examine the actions of $G$ on the spaces of holomorphic $k$-differentials and on the first homology. We show that when $G$ is not cyclic, the characters of these actions do not necessarily determine the topological type of the action of $G$ on $S$.
\end{abstract}

\section{INTRODUCTION}

Suppose the finite group $G$ acts as orientation preserving homeomorphisms of the oriented surface $S$. By varying the complex structure of the quotient surface $S / G$ and lifting to $S$, one should get all Riemann surfaces $S_{1}$ with a group $G_{1}$ of holomorphic homeomorphisms of the same topological type as the action of $G$ on $S$. That is, these are actions by $G$ on Riemann surfaces that are analytically deformable to each other. Such a family is determined by an equivalence class of epimorphisms $\psi: \Gamma \rightarrow G$, where $\Gamma$ is a group of Fuchsian type and the kernel of $\psi$ is isomorphic to the fundamental group of $S$. These families were studied in [3-8, 10-12]. In $\S 1$ we review the relevant facts from Teichmüller space theory which are needed to describe these families.

There are questions about these families that can be answered given $\psi$. For instance, is $G$ the automorphism group of the generic element of this family? If not, what is the automorphism group of the generic element? This is important to know if one is interested in the equisingularity strata of the singular set of the moduli space of genus $g$ [14]. Using a result of Singerman [18], we characterize in $\S 2$ those $G$-actions for which the normalizer of $G$ in the automorphism group

Received by the editors May 9, 1990 and, in revised form, October 15, 1990.

1991 Mathematics Subject Classification. Primary 14H15, 14H30, 14H45; Secondary 30F10. 
of any surface in the corresponding family is strictly larger than $G$. We also note that if generically the normalizer of $G$ is not the entire automorphism group, then the family consists of one point. Next we consider the set of all Riemann surfaces of genus $g$ with a subgroup of its automorphism group isomorphic to $G$. In $\S 3$ we see that for certain $G$ the components of this subvariety do not correspond in a one-to-one fashion with the topological actions of $G$, that is, there may be a $G$-action which determines a family completely contained in the family for another action of $G$.

Another set of questions involves invariants associated to group actions. If $G$ is contained in the automorphism group of the Riemann surface $S$, then there is an induced action of $G$ on the vector spaces of $k$-differentials on $S$ for all $k \geq 1$. Note that the representation on quadratic differentials of the full automorphism group of $S$ determines the singularity of the moduli space at $S$. The sequence of characters of these representations of $G$ is determined up to composition with automorphisms of $G$. These invariants could be used to decide whether or not two epimorphisms $\psi_{1}, \psi_{2}: \Gamma \rightarrow G$ determine equivalent actions of $G$. For cyclic groups these questions have been addressed by Guerrero [6], Harvey [7], A. Kuribayashi [10], and I. Kuribayashi [11]. In fact I. Kuribayashi proves that the sequence of characters determines the action of $G$ for $G$ cyclic. In $\S 4$ we offer some negative examples. We first review the relationship between the characters of the representations of $G$ on differentials and the rotation data at fixed points. We prove that the character of the representation of $G$ on integral homology determines the signature of $\Gamma$. Using the results of $\S 2$, we give two unramified $S_{7}$ covers of the surface of genus 2 , one of which inherits the hyperelliptic involution and the other does not. Since both covers are unramified, the Eichler trace formula [4] implies that the corresponding representations of $S_{7}$ on $k$-differentials are equivalent for all $k$. We also give two examples of unramified $D_{2 n}$ covers for even $n$. To show they are not equivalent, we calculate the representations on integral homology. We see that these representations are equivalent in $\operatorname{Sp}(2 g, \mathbb{Q})$, but not in $\operatorname{Sp}(2 g, \mathbb{Z})$. These examples should be contrasted with cyclic group actions yielding representations on integral homology that are equivalent in $\operatorname{SL}(2 g, \mathbb{Z})$, but not in $\operatorname{Sp}(2 g, \mathbb{Z})[5]$.

We state some results from $[3,7-10,12]$. We will include some details, since we will be making explicit calculations in later sections.

Fix a closed, oriented surface $S$ of genus $g$, with $g \geq 2$. Let $K=K_{g}$ denote $\pi_{1}(S, *)$, the fundamental group of $S$, Aut ${ }^{+}(K)$ the group of orientation preserving automorphisms of $K, \operatorname{Inn}(K)$ the subgroup of inner automorphisms of $K$, and $\operatorname{Mod}_{g}=\operatorname{Out}^{+}(K)=\operatorname{Aut}^{+}(K) / \operatorname{Inn}(K)$ the modular group or mapping class group of genus $g$. By a theorem of Nielsen this is isomorphic to the group of orientation preserving homeomorphisms of $S$ modulo isotopy. Suppose $\theta$ is an orientation preserving homeomorphism of $S$. It may not fix the basepoint, and so it may not induce a well-defined automorphism of $K$. But $\theta$ is isotopic to homeomorphisms that do fix the basepoint, and so $\theta$ induces a well-defined element of $\mathrm{Out}^{+}(K)$, denoted by $\bar{\theta}$. We use the same notation for groups of homeomorphisms of $S$. 
Suppose $S_{1}$ and $S_{2}$ are two Riemann surfaces of genus $g$ and for $i=$ $1,2, G_{i}<\operatorname{Aut}\left(S_{i}\right)$, the group of holomorphic homeomorphisms of $S_{i}$. We say $\left(S_{1}, G_{1}\right)$ is topologically equivalent to $\left(S_{2}, G_{2}\right)$ if there is an orientation preserving homeomorphism $\theta: S_{1} \rightarrow S_{2}$ and an isomorphism $\beta: G_{1} \rightarrow G_{2}$ such that for all $g \in G_{1}, \theta \circ g=\beta(g) \circ \theta$. Suppose $\pi_{i}: S_{i} \rightarrow S_{i} / G_{i}$ is the quotient map. Then this is equivalent to the existence of orientation preserving homeomorphisms $\theta: S_{1} \rightarrow S_{2}$ and $\theta_{0}: S_{1} / G_{1} \rightarrow S_{2} / G_{2}$ such that $\pi_{2} \circ \theta=$ $\theta_{0} \circ \pi_{1}$. If $\theta$ can be chosen to be biholomorphic, then we say $\left(S_{1}, G_{1}\right)$ is isomorphic to $\left(S_{2}, G_{2}\right)$.

Again suppose $G_{i}<\operatorname{Aut}\left(S_{i}\right)$ for $i=1,2$. Suppose $\theta_{i}: S \rightarrow S_{i}, i=1,2$, are any orientation preserving homeomorphisms. Then $G_{i}^{\prime}=\theta_{i}^{-1} G_{i} \theta_{i}, i=$ 1,2 , are two groups of homeomorphisms of $S$, which determine subgroups $\overline{G_{1}^{\prime}}$ and $\overline{G_{2}^{\prime}}$ of $\operatorname{Mod}_{g}$. If $\left(S_{1}, G_{1}\right)$ is topologically equivalent to $\left(S_{2}, G_{2}\right)$ by $\theta$, and $\alpha=\theta_{2}^{-1} \circ \theta \circ \theta_{1}$, then $\overline{G_{2}^{\prime}}=\bar{\alpha} \overline{G_{1}^{\prime}} \bar{\alpha}^{-1}$ and so $\overline{G_{1}^{\prime}}$ and $\overline{G_{2}^{\prime}}$ are conjugate subgroups of $\operatorname{Mod}_{g}$.

On the other hand, suppose $G$ is a finite subgroup of $\operatorname{Mod}_{g}$. This determines, up to isomorphism, a unique extension of $K$ via

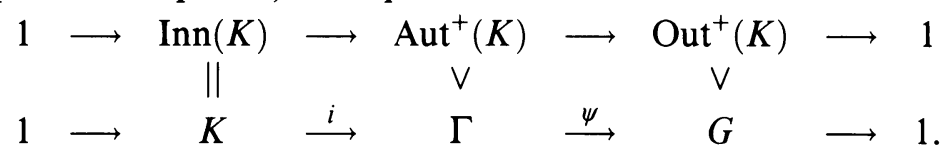

By Kerckhoff's solution of the Nielsen realization problem [9], $\Gamma$ is a group of Fuchsian type, say with signature $\left[p ; m_{1}, \ldots, m_{t}\right]$. Let $U$ be the upper half-plane. Then $\operatorname{PSL}(2, \mathbb{R})$ acts on $U$ as its group of holomorphic homeomorphisms. Let $T(\Gamma)$, the Teichmüller space of $\Gamma$, be one of the two components of the set of equivalence classes $[r]$ modulo conjugation by $\operatorname{PSL}(2, \mathbb{R})$ of injections $r: \Gamma \rightarrow \operatorname{PSL}(2, \mathbb{R})$ such that $r(\Gamma)$ is discrete. Let $\operatorname{Mod}(\Gamma)=$ $\operatorname{Aut}^{+}(\Gamma) / \operatorname{Inn}(\Gamma)$, where again Aut $^{+}(\Gamma)$ is the group of orientation preserving type preserving automorphisms of $\Gamma$. Suppose $\alpha \in \operatorname{Aut}^{+}(\Gamma)$. Then $\bar{\alpha} \in$ $\operatorname{Mod}(\Gamma)$ acts on $T(\Gamma)$ (on the right) by $\bar{\alpha}[r]=[r \circ \alpha]$. Then $\operatorname{Mod}(\Gamma)$ acts discontinuously on $T(\Gamma)$ and $\mathscr{M}(\Gamma)=T(\Gamma) / \operatorname{Mod}(\Gamma)$ is the space of conjugacy classes of discrete subgroups of $\operatorname{PSL}(2, \mathbb{R})$ isomorphic to $\Gamma$. The injection $i$ induces a map $\bar{i}: T(\Gamma) \rightarrow T(K)=T_{g}$, defined by $\bar{i}[r]=[r \circ i]$, which is a holomorphic homeomorphism onto $T_{g}^{G}$, the locus of fixed points of $G$ in $T_{g}$.

Suppose $[r] \in T(\Gamma)$ and let $\rho=r \circ i$. Let $S_{\rho}$ denote the Riemann surface $U / \rho(K)$ of genus $g$. Then $G$ acts on $S_{\rho}$ as follows. Let $z \in U$ and $g \in G$. Suppose $\gamma \in \Gamma$ is any element that projects to $g$. Then

$$
g \rho(K) z=r(\gamma) \rho(K) z .
$$

This gives us an injection $\phi_{r}: G \rightarrow \operatorname{Aut}\left(S_{\rho}\right)$. Let $G_{r}=\phi_{r}(G)$. By covering space theory there is a homeomorphism $\theta: S \rightarrow S_{\rho}$ inducing $\pi_{1}(S, *)=$ $K \stackrel{r}{\rightarrow} r(K) \simeq \pi_{1}\left(S_{\rho}, *\right)$, and the subgroup of $\mathrm{Out}^{+}(K)$ determined by $G_{r}$ and $\theta$ as above is $G$.

Let $S_{r}=U / r(\Gamma)$ and $\pi: S_{\rho} \rightarrow S_{r}$ be the quotient map by the action of $G_{r}$. Then $S_{r}$ is a Riemann surface of genus $p$, with $t$ distinguished points $p_{1}, \ldots, p_{t}$, which are the ramification points for the map $\pi$. If $\Gamma$ has presentation

$$
\begin{aligned}
\left\langle x_{1}, y_{1}, \ldots, y_{p}, z_{1}, \ldots, z_{t}\right|\left[x_{1}, y_{1}\right] \cdots & {\left[x_{p}, y_{p}\right] z_{1} \cdots z_{t} } \\
= & \left.1=z_{1}^{m_{1}}=\cdots=z_{t}^{m_{t}}\right\rangle,
\end{aligned}
$$


then

(a) $\psi\left(x_{1}\right), \psi\left(y_{1}\right), \ldots, \psi\left(z_{t}\right)$ generate $G$,

(b) $\psi\left(z_{i}\right)$ has order $m_{i}$ in $G$,

(c) the elements of $G_{r}$ which fix points of $S_{\rho}$ over $p_{i}$ are conjugate to powers of $\phi_{r}\left(\psi\left(z_{i}\right)\right)$,

(d) (the Riemann-Hurwitz relation)

$$
2 g-2=|G|\left(2 p-2+\sum_{i=1}^{t}\left(1-\frac{1}{m_{i}}\right)\right) .
$$

Now suppose $[r]$ and $[s]$ are two elements of $T(\Gamma)$. Since $r(\Gamma)$ is isomorphic to $s(\Gamma)$ and they have compact quotient, it is known that there is a homeomorphism $\theta$ of $U$ such that for all $\gamma \in \Gamma$,

$$
s(\gamma) \circ \theta=\theta \circ r(\gamma) \text {. }
$$

Since $[r]$ and $[s]$ lie in the same component of $T(\Gamma), \theta$ is orientation preserving [12]. Let $\rho=r \circ i$ and $\sigma=s \circ i$. Then $\theta$ descends to an orientation preserving homeomorphism of $S_{\rho}$ and $S_{\sigma}$ intertwining the actions of $G_{r}$ and $G_{s}$. Thus $\left(S_{\rho}, G_{r}\right)$ is topologically equivalent to $\left(S_{\sigma}, G_{s}\right)$.

Suppose $\alpha \in \operatorname{Aut}^{+}(K)$ and consider $\bar{\alpha} G \bar{\alpha}^{-1}$. Then $\alpha \Gamma \alpha^{-1}$ is the subgroup of Aut ${ }^{+}(K)$ containing $\operatorname{Inn}(K)$ which projects to $\bar{\alpha} G \bar{\alpha}^{-1}$. We denote by $l_{\alpha}$ the isomorphism of conjugation by $\alpha, l_{\alpha}(\gamma)=\alpha \gamma \alpha^{-1}$. Then we have the followng commutative diagram:

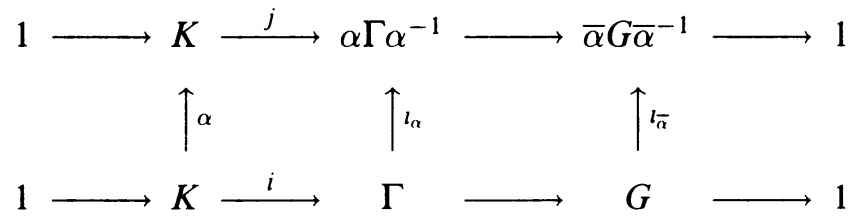

Then $\bar{l}_{\alpha}: T\left(\alpha \Gamma \alpha^{-1}\right) \rightarrow T(\Gamma)$ is an analytic homeomorphism and we have $\bar{j} T\left(\alpha \Gamma \alpha^{-1}\right)=T_{g}^{\bar{\alpha} G \bar{\alpha}^{-1}}=\bar{\alpha}^{-1}\left(T_{g}^{G}\right)$. Note that if $\alpha \in \Gamma$, then $\bar{l}_{\alpha}$ is the identity map on $T(\Gamma)$.

We can draw several conclusions from this. First let $N(G)$ be the normalizer of $G$ in $\operatorname{Mod}_{g}$. Then $N(G)$ is contained in the stabilizer of $\bar{i} T(\Gamma)=T_{g}^{G}$ in $\operatorname{Mod}_{g}$ and the homomorphism which sends $\alpha$ to $l_{\alpha}$ induces an injection of $N(G) / G$ into $\operatorname{Mod}(\Gamma)$. Since $K \triangleleft \Gamma$, we can define the relative mapping class group of $\Gamma$ and $K$ as

$$
\operatorname{Mod}(\Gamma, K)=\{\bar{\alpha} \in \operatorname{Mod}(\Gamma) \mid \alpha(K)=K\}
$$

Then $\operatorname{Mod}(\Gamma, K)$ is a subgroup of finite index in $\operatorname{Mod}(\Gamma)$ and

$$
N(G) / G \simeq \operatorname{Mod}(\Gamma, K)
$$

On the other hand, if $\bar{\alpha}\left(T_{g}^{G}\right)=T_{g}^{G}$, then $T_{g}^{\bar{\alpha} G \bar{\alpha}^{-1}}=T_{g}^{G}$. Let $H$ be the group generated by $\bar{\alpha} G \bar{\alpha}^{-1}$ for $\bar{\alpha}$ in the stabilizer of $T_{g}^{G}$. Then $T_{g}^{G}=T_{g}^{H}$ and so $H$ is a finite group. Thus $N(G)$ is a subgroup of finite index in $N(H)$, which is the stabilizer of $T_{g}^{G}$ in $\operatorname{Mod}_{g}$.

Suppose $\alpha \in \operatorname{Aut}^{+}(K)$ and $[r] \in T\left(\alpha \Gamma \alpha^{-1}\right)$. Then $\bar{l}_{\alpha}[r]=\left[r \circ l_{\alpha}\right] \in T(\Gamma)$. Let $\rho_{1}=r \circ j$ and $\rho_{2}=r \circ l_{\alpha} \circ i$. Then $\rho_{1} \circ \alpha=\rho_{2}$ and so $\rho_{1}(K)=\rho_{2}(K)$, $S_{\rho_{1}}=S_{\rho_{2}}$, and $\phi_{r}\left(\bar{\alpha} G \bar{\alpha}^{-1}\right)=\phi_{r l_{l}}(G)$ in $\operatorname{Aut}\left(S_{\rho_{1}}\right)$. 
We can summarize the above remarks as follows. Suppose $\mathscr{G}$ is a conjugacy class of finite subgroups of $\operatorname{Mod}_{g}$ and let $G \in \mathscr{G}$. We also denote this conjugacy class by $[G]$. Let $\Gamma$ be the subgroup of Aut $^{+}(K)$ which projects to $G$. Then $\Gamma$ is of Fuchsian type and

$$
\mathscr{M}(\mathscr{G})=T(\Gamma) / \operatorname{Mod}(\Gamma, K)
$$

is the moduli space of isomorphism classes of pairs $\left(S_{1}, G_{1}\right)$, where $S_{1}$ is a Riemann surface of genus $g, G_{1}<\operatorname{Aut}\left(S_{1}\right)$, and $\bar{G}_{1}$, any subgroup of $\operatorname{Mod}_{g}$ induced by $G_{1}$, lies in $\mathscr{G}$. The map $\bar{i}: T(\Gamma) \rightarrow T_{g}$ covers the natural forgetful map $\mathscr{M}(\mathscr{G}) \rightarrow \mathscr{M}_{g}$ which sends $\left(S_{1}, G_{1}\right)$ to $S_{1}$. Let $\mathscr{M}_{g}^{\mathscr{G}}$ denote the image of this map. This map is finite-to-one, but may not be injective since the submanifolds $T_{g}^{G}$ and $T_{g}^{\bar{\alpha} G \bar{\alpha}^{-1}}$ of $T_{g}$ may not be disjoint, that is, there may be a finite subgroup $H$ of $\operatorname{Mod}_{g}$ which contains $G$ and $\bar{\alpha} G \bar{\alpha}^{-1}$ but these are not conjugate in $H$. For example, using the results of the next section, we can see that there is only one conjugacy class $\mathscr{G}$ of subgroups of $\operatorname{Mod}_{5}$ isomorphic to $S_{4}$ and the map from $\mathscr{M}(\mathscr{G})$ to $\mathscr{M}_{5}^{\mathscr{G}}$ is generically two-to-one. If $\Gamma$ has signature $\left[p ; m_{1}, \ldots, m_{t}\right]$, then

$$
\operatorname{dim} \mathscr{M}(\mathscr{G})=\operatorname{dim} T(\Gamma)=3 p-3+t .
$$

Finally suppose $\Gamma$ is a group of Fuchsian type with signature $\left[p ; m_{1}, \ldots, m_{t}\right]$ and $\psi: \Gamma \rightarrow G$ is a homomorphism to the finite group $G$ having the above properties (a), (b), and (d) for some $g \geq 2$. Then the kernel of $\psi$ is torsion free and isomorphic to $K$. Let $i: K \rightarrow \Gamma$ be an isomorphism from $K$ to ker $\psi$. Since the center of $\Gamma$ is trivial, the action of $\Gamma$ on $K$ induced by conjugation yields injections $\Gamma \rightarrow \operatorname{Aut}^{+}(K)$ and $G \rightarrow \operatorname{Mod}_{g}$ and we are in the situation above. More precisely, suppose $\gamma \in \Gamma$ and let $\alpha_{\gamma}$ denote the automorphism of $K$ induced by conjugation by $\gamma$, that is, $l_{\gamma} \circ i=i \circ \alpha_{\gamma}$. Note that if $\gamma=i(k)$ for $k \in K$, then $\alpha_{\gamma}=l_{k}$. For $a \in G$, let $\sigma(a)$ denote the induced element of $\operatorname{Mod}_{g}$, that is, if $\gamma \in \Gamma$ and $\psi(\gamma)=a$, then $\sigma(a)=\bar{\alpha}_{\gamma}$. We say that $\sigma$ is induced by the pair $(i, \psi)$. Then the following can easily be shown:

(a) If $\delta \in \operatorname{Aut}(G)$, then $(i, \delta \circ \psi)$ induces $\sigma \circ \delta^{-1}$.

(b) If $\beta \in \operatorname{Aut}^{+}(\Gamma)$, then $\left(\beta^{-1} \circ i, \psi \circ \beta\right)$ induces $\sigma$.

(c) If $\alpha \in \operatorname{Aut}^{+}(K)$, then $\left(i \circ \alpha^{-1}, \psi\right)$ induces $l_{\bar{\alpha}} \circ \sigma$.

(d) If $\beta \in \operatorname{Aut}^{+}(\Gamma)$ and preserves $i(K)$, then there is a $\delta \in \operatorname{Aut}(G)$ so that $\delta \circ \psi \circ \beta=\psi$ and there is an $\alpha \in \operatorname{Aut}^{+}(K)$ so that $\beta \circ i \circ \alpha=i$. Then $(i, \psi \circ \beta)$ and $(\beta \circ i, \psi)$ induce $\sigma \circ \delta=l_{\bar{\alpha}} \circ \sigma$ and hence $\bar{\alpha} \in N(\sigma(G))$.

Thus $\sigma(G)$, the image of $G$ in $\operatorname{Mod}_{g}$, depends on both $i$ and $\psi$, but the conjugacy class $\mathscr{G}_{\psi}=[\sigma(G)]$ is determined by $\psi$. Note that two such epimorphisms $\psi_{1}, \psi_{2}: \Gamma \rightarrow G$ determine isomorphic extensions of $K$ and hence topologically equivalent $G$-actions iff there are $\beta \in \operatorname{Aut}^{+}(\Gamma)$ and $\lambda \in$ $\operatorname{Aut}(G)$ so that $\psi_{2}=\lambda \circ \psi_{1} \circ \beta$.

Notation. Suppose $\mathscr{G}$ and $\mathscr{H}$ are conjugacy classes of subgroups of $\operatorname{Mod}_{g}$. Then we will write $\mathscr{G}<\mathscr{H}$ (resp. $\mathscr{G} \triangleleft \mathscr{H}$ ) if there are $G \in \mathscr{G}$ and $H \in \mathscr{H}$ such that $G<H$ (resp. $G \triangleleft H)$.

In this paper we want to study the subvarieties $\mathscr{M}_{g}^{\mathscr{G}}$ of $\mathscr{M}_{g}$. We have already noted their dimensions. Their singularities would have similar description as for 
itself, possible quotient singularities at $S_{1}$ when $G_{1} \neq N_{\operatorname{Aut}\left(S_{1}\right)}\left(G_{1}\right)$, but also possible identification singularities when $\operatorname{Aut}\left(S_{1}\right)$ contains two subgroups $G_{1}$ and $G_{2}$ from $\mathscr{G}$ which are not conjugate in $\operatorname{Aut}\left(S_{1}\right)$.

It is clear that if $G<H$ are finite subgroups of $\operatorname{Mod}_{g}$, then $\mathscr{M}_{g}^{[H]} \subseteq \mathscr{M}_{g}^{[G]}$. We will first examine cases when these can be equal even though $G \neq H$. If $\psi: \Gamma \rightarrow G$ and $\Gamma$ has presentation (2), let $a_{i}=\psi\left(x_{i}\right), b_{i}=\psi\left(y_{i}\right), 1 \leq i \leq p$, and $c_{i}=\psi\left(z_{i}\right), 1 \leq i \leq t$.

Theorem. Let $\psi: \Gamma \rightarrow G$ and $i: K_{g} \rightarrow \Gamma$ induce the inclusion of $G$ as $a$ subgroup of $\operatorname{Mod}_{g}$. Suppose one of the following is true:

1. $\Gamma$ has signature $[2 ;-]$ and there is an automorphism $\alpha$ of $G$ such that, if $d=a_{1}^{-1} b_{1}^{-1} a_{2} b_{2}$,

$$
\alpha\left(a_{1}\right)=a_{1}^{-1}, \quad \alpha\left(b_{1}\right)=b_{1}^{-1}, \quad \alpha\left(a_{2}\right)=d a_{2}^{-1} d^{-1}, \quad \alpha\left(b_{2}\right)=d b_{2}^{-1} d^{-1} .
$$

2. $\Gamma$ has signature $[1 ; k, k]$ and there is an $\alpha \in \operatorname{Aut}(G)$ such that, if $d=a_{1}^{-1} b_{1}^{-1} c_{1}$,

$$
\alpha\left(a_{1}\right)=a_{1}^{-1}, \quad \alpha\left(b_{1}\right)=b_{1}^{-1}, \quad \alpha\left(c_{1}\right)=d c_{2} d^{-1}, \quad \alpha\left(c_{2}\right)=d c_{1} d^{-1} .
$$

3. $\Gamma$ has signature $[1 ; k]$ and there is an $\alpha \in \operatorname{Aut}(G)$ such that

$$
\alpha\left(a_{1}\right)=a_{1}^{-1}, \quad \alpha\left(b_{1}\right)=b_{1}^{-1}, \quad \alpha\left(c_{1}\right)=a_{1}^{-1} b_{1}^{-1} c_{1} b_{1} a_{1} .
$$

4. $\Gamma$ has signature $[0 ; k, l, k, l]$ for some $k, l$ with $2 \leq k$ and $3 \leq l$. If $k \neq l$, there is an $\alpha \in \operatorname{Aut}(G)$ such that

$$
\alpha\left(c_{1}\right)=c_{3}, \quad \alpha\left(c_{2}\right)=c_{4}, \quad \alpha\left(c_{3}\right)=c_{1}, \quad \alpha\left(c_{4}\right)=c_{2} .
$$

If $k=l$, then $\alpha$ exists after replacing $\psi$ by $\psi \circ \mu$ for some $\mu \in \operatorname{Aut}^{+}(\Gamma)$.

5. $\Gamma$ has signature $[0 ; k, k, k, k]$ for some $k$ with $3 \leq k$ and there are $\alpha, \beta \in \operatorname{Aut}(G)$ such that

$$
\begin{gathered}
\alpha\left(c_{1}\right)=c_{3}, \quad \alpha\left(c_{2}\right)=c_{4}, \quad \alpha\left(c_{3}\right)=c_{1}, \quad \alpha\left(c_{4}\right)=c_{2}, \\
\beta\left(c_{1}\right)=c_{2}, \quad \beta\left(c_{2}\right)=c_{1}, \quad \beta\left(c_{3}\right)=c_{1}^{-1} c_{4} c_{1}, \quad \beta\left(c_{4}\right)=c_{2} c_{3} c_{2}^{-1} .
\end{gathered}
$$

6. $\Gamma$ has signature $[0 ; l, l, k]$ for some $k, l$ with $3 \leq l, 2 \leq k$ and at least one of the inequalities is strict. If $k \neq l$, there is an $\alpha \in \operatorname{Aut}(G)$ such that

$$
\alpha\left(c_{1}\right)=c_{2}, \quad \alpha\left(c_{2}\right)=c_{1}, \quad \alpha\left(c_{3}\right)=c_{2} c_{3} c_{2}^{-1} .
$$

If $k=l$, then $\alpha$ exists after replacing $\psi$ by $\psi \circ \mu$ for some $\mu \in \operatorname{Aut}^{+}(\Gamma)$.

7. $\Gamma$ has signature $[0 ; k, k, k]$ for some $k \geq 4$ and there is $a \beta \in \operatorname{Aut}(G)$ such that

$$
\beta\left(c_{1}\right)=c_{2}, \quad \beta\left(c_{2}\right)=c_{3}, \quad \beta\left(c_{3}\right)=c_{1} .
$$

8. $\Gamma$ has signature $[0 ; k, k, k]$ for some $k \geq 4$ and there are $\alpha, \beta \in \operatorname{Aut}(G)$ such that

$$
\begin{gathered}
\alpha\left(c_{1}\right)=c_{2}, \quad \alpha\left(c_{2}\right)=c_{1}, \quad \alpha\left(c_{3}\right)=c_{2} c_{3} c_{2}^{-1}, \\
\beta\left(c_{1}\right)=c_{2}, \quad \beta\left(c_{2}\right)=c_{3}, \quad \beta\left(c_{3}\right)=c_{1} .
\end{gathered}
$$

If $H$ is the corresponding group with presentation

$1,2,3,4,6 . \quad H=\langle G, a| \cdots, a^{2}=1, a g a=\alpha(g)$, for $\left.g \in G\right\rangle$,

5. $H=\langle G, a, b| \cdots, a^{2}=b^{2}=1, a g a=\alpha(g), b g b=\beta(g)$, for $\left.g \in G, a b a b=\left(c_{2} c_{3}\right)^{-1}\right\rangle$, 
7. $H=\langle G, b| \cdots, b^{3}=1, b g b^{2}=\beta(g)$, for $\left.g \in G\right\rangle$,

8. $H=\langle G, a, b| \cdots, a^{2}=b^{3}=1, a g a=\alpha(g), b g b^{2}=\beta(g)$, for $\left.g \in G, a b a b=c_{1}^{-1}\right\rangle$,

where the dots denote the relations of $G$, then there exist $\Gamma_{0}$, an inclusion $j$ : $\Gamma \rightarrow \Gamma_{0}$, and an epimorphism $\psi_{0}: \Gamma_{0} \rightarrow H$ such that $\psi=\psi_{0} \circ j$ and $\left(j \circ i, \psi_{0}\right)$ induces the inclusion of $H$ as a subgroup of $\operatorname{Mod}_{g}$ with the property that $G \neq$ $H, G \triangleleft H$ and $\mathscr{M}_{g}^{[G]}=\mathscr{M}_{g}^{[H]}$.

Conversely, any subgroup $H$ of $\operatorname{Mod}_{g}$ with the above property arises in this way.

Proof. By the above remarks $\Gamma$ is identified with a subgroup of $\operatorname{Aut}^{+}(K)$ and $\psi$ becomes the projection in (1). Suppose there is a finite subgroup $H$ of $\operatorname{Mod}_{g}$ such that $G \triangleleft H, G \neq H$, and $\mathscr{M}_{g}^{[G]}=\mathscr{M}_{g}^{[H]}$. Let $\Gamma_{0}$ be the subgroup of $\operatorname{Aut}^{+}(K)$ containing $\operatorname{Inn}(K)$ which projects to $H$ and let $\psi_{0}: \Gamma_{0} \rightarrow H$ be the projection. Then $\Gamma \triangleleft \Gamma_{0}$ and, if $j: \Gamma \rightarrow \Gamma_{0}$ denotes the inclusion, then $\bar{j} T\left(\Gamma_{0}\right)=T(\Gamma)$, since they have the same dimension.

Assume $\Gamma_{0}$ has signature $\left[p_{0} ; n_{1}, \ldots, n_{s}\right]$ and presentation

$$
\begin{aligned}
\left\langle X_{1}, Y_{1}, \ldots, Y_{p_{0}}, Z_{1}, \ldots, Z_{s}\right|\left[X_{1}, Y_{1}\right] \cdots\left[X_{p_{0}}, Y_{p_{0}}\right] Z_{1} \cdots Z_{s} & \\
& \left.=1=Z_{1}^{n_{1}}=\cdots=Z_{s}^{n_{s}}\right\rangle,
\end{aligned}
$$

and $\Gamma$ has signature $\left[p ; n_{1}, \ldots, n_{t}\right]$ and presentation (2). Singerman lists all possible pairs $\Gamma, \Gamma_{0}$ with the above properties in [18]. Let $H_{0}=\Gamma_{0} / \Gamma$ and let $\theta_{0}: \Gamma_{0} \rightarrow H_{0}$ be the quotient homomorphism. Then $\left(j, \theta_{0}\right)$ induces $H_{0}<\operatorname{Mod}(\Gamma)$. Given $\psi$ so that $\operatorname{ker} \psi=i(K)$, then $i(K)$ will be a normal subgroup of $\Gamma_{0}$ iff $H_{0}<\operatorname{Mod}(\Gamma, K)$ iff for all $\gamma \in \Gamma_{0}$ there is an $\alpha_{\gamma} \in \operatorname{Aut}(G)$ so that $\psi \circ l_{\gamma}=\alpha_{\gamma} \circ \psi$. If this is true, then $H_{0} \simeq H / G$. So, for each generator of $H_{0}$, we choose a particular coset representative $\gamma \in \Gamma_{0}$ projecting to it. Then $\psi_{0}(\gamma)$ gives a generator of $H$ over $G$ so that $l_{\psi_{0}(\gamma)}=\alpha_{\gamma} \in \operatorname{Aut}(G)$ and any word in these $\gamma$ projecting to the identity in $H_{0}$ must lie in $\Gamma$ and so project via $\psi$ into $G$. The following generators and relations determine an inclusion $j$ of $\Gamma$ as a subgroup of $\Gamma_{0}$ and can be verified using a standard Reidemeister-Schreier rewriting process [13].

1. $\Gamma$ has signature $[2 ;-], \Gamma_{0}$ has signature $[0 ; 2,2,2,2,2,2], H_{0}=$ $\mathbb{Z}_{2}=\{ \pm 1\}$, and $\theta_{0}\left(Z_{i}\right)=-1,1 \leq i \leq 6$. We identify $\operatorname{ker} \theta_{0}$ with $\Gamma$ by

$$
x_{1}=Z_{1} Z_{2}, \quad y_{1}=Z_{3} Z_{2}, \quad x_{2}=Z_{4} Z_{5}, \quad y_{2}=Z_{6} Z_{5} .
$$

If we take $\gamma=Z_{2}$, then $\gamma^{2}=1$ and $\alpha=\alpha_{\gamma}$. Since $x_{1}, y_{1}, x_{2}, y_{2}, Z_{2}$ generate $\Gamma_{0}$, we can calculate $\psi_{0}$. Setting $a=\psi_{0}\left(Z_{2}\right)$ and $d=a_{1}^{-1} b_{1}^{-1} a_{2} b_{2}$, we get

$$
\begin{aligned}
& \psi_{0}\left(Z_{1}\right)=a_{1} a, \quad \psi_{0}\left(Z_{2}\right)=a, \quad \psi_{0}\left(Z_{3}\right)=b_{1} a, \\
& \psi_{0}\left(Z_{4}\right)=a_{2} d^{-1} a, \quad \psi_{0}\left(Z_{5}\right)=d^{-1} a, \quad \psi_{0}\left(Z_{6}\right)=b_{2} d^{-1} a .
\end{aligned}
$$

2. $\Gamma$ has signature $[1 ; k, k], \Gamma_{0}$ has signature $[0 ; 2,2,2,2, k], H_{0}=\mathbb{Z}_{2}$, $\theta_{0}\left(Z_{i}\right)=-1,1 \leq i \leq 4$, and $\theta_{0}\left(Z_{5}\right)=1$. We identify $\operatorname{ker} \theta_{0}$ with $\Gamma$ by

$$
x_{1}=Z_{2} Z_{3}, \quad y_{1}=Z_{4} Z_{3}, \quad z_{1}=Z_{5}, \quad z_{2}=Z_{1} Z_{5} Z_{1} .
$$

If we take $\gamma=Z_{3}$, then $\gamma^{2}=1$ and $\alpha=\alpha_{\gamma}$. Setting $a=\psi_{0}\left(Z_{3}\right)$ and 
$d=a_{1}^{-1} b_{1}^{-1} c_{1}$, we get

$$
\begin{gathered}
\psi_{0}\left(Z_{1}\right)=d^{-1} a, \quad \psi_{0}\left(Z_{2}\right)=a_{1} a, \quad \psi_{0}\left(Z_{3}\right)=a, \\
\psi_{0}\left(Z_{4}\right)=b_{1} a, \quad \psi_{0}\left(Z_{5}\right)=c_{1} .
\end{gathered}
$$

3. $\Gamma$ has signature $[1 ; k], \Gamma_{0}$ has signature $[0 ; 2,2,2,2 k], H_{0}=\mathbb{Z}_{2}$, and $\theta_{0}\left(Z_{i}\right)=-1,1 \leq i \leq 4$. We identify $\operatorname{ker} \theta_{0}$ with $\Gamma$ by

$$
x_{1}=Z_{1} Z_{2}, \quad y_{1}=Z_{3} Z_{1}, \quad z_{1}=Z_{4}^{2} .
$$

If we take $\gamma=Z_{1}$, then $\gamma^{2}=1$ and $\alpha=\alpha_{\gamma}$. Setting $a=\psi_{0}\left(Z_{1}\right)$, we get

$$
\psi_{0}\left(Z_{1}\right)=a, \quad \psi_{0}\left(Z_{2}\right)=a a_{1}, \quad \psi_{0}\left(Z_{3}\right)=a b_{1}^{-1}, \quad \psi_{0}\left(Z_{4}\right)=a b_{1}^{-1} a_{1}^{-1} .
$$

4. $\Gamma$ has signature $[0 ; k, l, k, l], \Gamma_{0}$ has signature $[0 ; 2,2, k, l], H_{0}=$ $\mathbb{Z}_{2}, \theta_{0}\left(Z_{i}\right)=-1,1 \leq i \leq 2$, and $\theta_{0}\left(Z_{i}\right)=1,3 \leq i \leq 4$. We identify $\operatorname{ker} \theta_{0}$ with $\Gamma$ by

$$
z_{1}=Z_{3}, \quad z_{2}=Z_{4}, \quad z_{3}=Z_{1} Z_{3} Z_{1}, \quad z_{4}=Z_{1} Z_{4} Z_{1} .
$$

If we take $\gamma=Z_{1}$, then $\gamma^{2}=1$ and $\alpha=\alpha_{\gamma}$. Setting $a=\psi_{0}\left(Z_{1}\right)$, we get

$$
\psi_{0}\left(Z_{1}\right)=a, \quad \psi_{0}\left(Z_{2}\right)=a c_{2}^{-1} c_{1}^{-1}, \quad \psi_{0}\left(Z_{3}\right)=c_{1}, \quad \psi_{0}\left(Z_{4}\right)=c_{2} .
$$

5. $\Gamma$ has signature $[0 ; k, k, k, k], \Gamma_{0}$ has signature $[0 ; 2,2,2, k], H_{0}=$ $\mathbb{Z}_{2} \times \mathbb{Z}_{2}=\left\{1, \varepsilon_{1}, \varepsilon_{2}, \varepsilon_{1} \varepsilon_{2}\right\}, \theta_{0}\left(Z_{1}\right)=\varepsilon_{1}, \theta_{0}\left(Z_{2}\right)=\varepsilon_{2}, \theta_{0}\left(Z_{3}\right)=\varepsilon_{1} \varepsilon_{2}$, and $\theta_{0}\left(Z_{4}\right)=1$. We identify $\operatorname{ker} \theta_{0}$ with $\Gamma$ by

$$
z_{1}=Z_{4}, \quad z_{2}=Z_{1} Z_{4} Z_{1}, \quad z_{3}=Z_{3} Z_{4} Z_{3}, \quad z_{4}=Z_{3} Z_{1} Z_{4} Z_{1} Z_{3} .
$$

If we take $\gamma_{1}=Z_{3}$ and $\gamma_{2}=Z_{1}$, then $\gamma_{1}^{2}=\gamma_{2}^{2}=1, \gamma_{1} \gamma_{2} \gamma_{1} \gamma_{2}=\left(z_{2} z_{3}\right)^{-1}$, $\alpha=\alpha_{\gamma_{1}}$, and $\beta=\alpha_{\gamma_{2}}$. Setting $a=\psi_{0}\left(Z_{3}\right)$ and $b=\psi_{0}\left(Z_{1}\right)$, we get

$$
\psi_{0}\left(Z_{1}\right)=b, \quad \psi_{0}\left(Z_{2}\right)=b c_{1}^{-1} a, \quad \psi_{0}\left(Z_{3}\right)=a, \quad \psi\left(Z_{4}\right)=c_{1} .
$$

6. $\Gamma$ has signature $[0 ; l, l, k], \Gamma_{0}$ has signature $[0 ; 2, l, 2 k], H_{0}=\mathbb{Z}_{2}$, $\theta_{0}\left(Z_{1}\right)=-1, \theta_{0}\left(Z_{2}\right)=1$, and $\theta_{0}\left(Z_{3}\right)=-1$. We identify $\operatorname{ker} \theta_{0}$ with $\Gamma$ by

$$
z_{1}=Z_{1} Z_{2} Z_{1}, \quad z_{2}=Z_{2}, \quad z_{3}=Z_{3}^{2} .
$$

If we take $\gamma=Z_{1}$, then $\gamma^{2}=1$ and $\alpha=\alpha_{\gamma}$. Setting $a=\psi_{0}\left(Z_{1}\right)$, we get

$$
\psi_{0}\left(Z_{1}\right)=a, \quad \psi_{0}\left(Z_{2}\right)=c_{2}, \quad \psi_{0}\left(Z_{3}\right)=a c_{1}^{-1} .
$$

7. $\Gamma$ has signature $[0 ; k, k, k], \Gamma_{0}$ has signature $[0 ; 3,3, k], H_{0}=\mathbb{Z}_{3}=$ $\left\{1, \varepsilon, \varepsilon^{2}\right\}, \theta_{0}\left(Z_{1}\right)=\varepsilon, \theta_{0}\left(Z_{2}\right)=\varepsilon^{2}$, and $\theta_{0}\left(Z_{3}\right)=1$. We identify $\operatorname{ker} \theta_{0}$ with $\Gamma$ by

$$
z_{1}=Z_{3}, \quad z_{2}=Z_{2} Z_{3} Z_{2}^{2}, \quad z_{3}=Z_{2}^{2} Z_{3} Z_{2} .
$$

If we take $\gamma=Z_{2}$, then $\gamma^{3}=1$ and $\beta=\alpha_{\gamma}$. Setting $b=\psi_{0}\left(Z_{2}\right)$, we get

$$
\psi_{0}\left(Z_{1}\right)=c_{1}^{-1} b^{2}, \quad \psi_{0}\left(Z_{2}\right)=b, \quad \psi_{0}\left(Z_{3}\right)=c_{1} .
$$

8. $\Gamma$ has signature $[0 ; k, k, k], \Gamma_{0}$ has signature $[0 ; 2,3,2 k], H_{0}=S_{3}$, $\theta_{0}\left(Z_{1}\right)=(12), \theta_{0}\left(Z_{2}\right)=(123)$, and $\theta_{0}\left(Z_{3}\right)=(13)$. We identify $\operatorname{ker} \theta_{0}$ with $\Gamma$ by

$$
z_{1}=Z_{3}^{2}, \quad z_{2}=Z_{2} Z_{3}^{2} Z_{2}^{2}, \quad z_{3}=Z_{2}^{2} Z_{3}^{2} Z_{2} .
$$


If we take $\gamma_{1}=Z_{1}$ and $\gamma_{2}=Z_{2}$, then $\gamma_{1}^{2}=\gamma_{2}^{3}=1, \gamma_{1} \gamma_{2} \gamma_{1} \gamma_{2}=z_{1}^{-1}, \alpha=\alpha_{\gamma_{1}}$, and $\beta=\alpha_{\gamma_{2}}$. Setting $a=\psi_{0}\left(Z_{1}\right)$ and $b=\psi_{0}\left(Z_{2}\right)$, we get

$$
\psi_{0}\left(Z_{1}\right)=a, \quad \psi_{0}\left(Z_{2}\right)=b, \quad \psi\left(Z_{3}\right)=b^{2} a .
$$

Moreover, with this particular $j$ and a presentation of the corresponding group $H_{0}$, the presentation of $H$ is that given in the theorem.

The remaining problem is that the generators of $\Gamma$ singled out above may not be the initially chosen ones, that is, suppose $j$ is replaced by $j \circ \mu^{-1}$ for some $\mu \in \operatorname{Aut}^{+}(\Gamma)$. Then by the remarks in $\S 1, H_{0}$ is replaced by $\bar{\mu} H_{0} \bar{\mu}^{-1}$. But $T(\Gamma)^{H_{0}}=\bar{j} T\left(\Gamma_{0}\right)=T(\Gamma)$ and so also $T(\Gamma)^{\bar{\mu} H_{0} \bar{\mu}^{-1}}=T(\Gamma)$. Let $H_{1}$ be the subgroup of $\operatorname{Mod}(\Gamma)$ generated by $\bar{\mu} H_{0} \bar{\mu}^{-1}$ for all $\bar{\mu} \in \operatorname{Mod}(\Gamma)$. Then $H_{0}<H_{1} \triangleleft \operatorname{Mod}(\Gamma)$ and $T(\Gamma)^{H_{1}}=T(\Gamma)$. Thus $H_{1}$ is a finite group and if $\Gamma_{1}$ is the subgroup of $\operatorname{Aut}^{+}(\Gamma)$ containing $\Gamma$ (i.e., $\operatorname{Inn}(\Gamma)$ ) which projects to $H_{1}$, then $\Gamma \triangleleft \Gamma_{1}$ and $\operatorname{dim} T(\Gamma)=\operatorname{dim} T\left(\Gamma_{1}\right)$. Therefore the pair $\Gamma, \Gamma_{1}$ must be on Singerman's list. Thus in all cases except 4 and 6 with $k=l$, we have $\Gamma_{0}=\Gamma_{1}$ and $H_{0}=H_{1}$. This shows that the results above are actually independent of $j$ for these cases. But in case 4 (resp. case 6) when $k=l$, the pair $\Gamma, \Gamma_{1}$ comes from case 5 (resp. case 8) and $H_{1} \simeq \mathbb{Z}_{2} \times \mathbb{Z}_{2}$ (resp. $\left.S_{3}\right)$. The three subgroups of $H_{1}$ isomorphic to $H_{0}$ are conjugate in $\operatorname{Mod}(\Gamma)$ and so there exists $\mu$ transforming our given $j$ from the beginning of the proof into the one of the above computations. This will replace $(i, \psi)$ with $\left(\mu^{-1} \circ i, \psi \circ \mu\right)$ which induces the same inclusion of $G$ into $\operatorname{Mod}_{g}$. Then $\psi \circ \mu$ has the property stated in the theorem.

Conversely, suppose $\psi$ satisfies any of the cases of the theorem. If we form the indicated group $H$, then by the calculations in the first part of the proof, there is an inclusion $j$ of $\Gamma$ as a normal subgroup of the corresponding $\Gamma_{0}$ and an epimorphism $\psi_{0}: \Gamma_{0} \rightarrow H$ such that $\psi_{0} \circ j=\psi$. Then $\left(j \circ i, \psi_{0}\right)^{\circ}$ induces an inclusion of $H$ as a subgroup of $\operatorname{Mod}_{g}$ such that $G \triangleleft H, G \neq H$, and, since $\bar{j} T\left(\Gamma_{0}\right)=T(\Gamma)$, we have $\mathscr{M}_{g}^{[G]}=\mathscr{M}_{g}^{[H]}$.

Remark. Suppose $G$ is a finite subgroup of $\operatorname{Mod}_{g}$ and suppose there is a subgroup $H$ of $\operatorname{Mod}_{g}$ with the properties

$$
G \triangleleft H, \quad G \neq H, \quad \text { and } \quad \mathscr{M}_{g}^{[G]}=\mathscr{M}_{g}^{[H]} .
$$

If $H_{1}$ is the subgroup of $\operatorname{Mod}_{g}$ generated by all such $H$, then $H_{1}$ also has the above property. It follows from the theorem that if $H_{1}$ corresponds to case $1,2,3,4,6$, or 7 , then $H_{1}$ is the only subgroup of $\operatorname{Mod}_{g}$ with the above property. On the other hand, if $H_{1}$ corresponds to case 5 (resp. case 8), then there are also three subgroups of $H_{1}$ corresponding to case 4 with $k=l$ (resp. three subgroups of $H_{1}$ corresponding to case 6 with $k=l$ and a subgroup corresponding to case 7 ) with the above property. Note that their conjugacy classes may or may not be distinct.

As was noted earlier, $\mathscr{M}(\Gamma)$ is the space of isomorphism classes of Riemann surfaces of genus $p$ with $t$ distinguished points $p_{1}, \ldots, p_{t}$ of orders $m_{1}, \ldots, m_{t}$ resp. For most $\Gamma$ the generic pointed Riemann surface corresponding to an element of $\mathscr{M}(\Gamma)$ has trivial automorphism group. But for the $\Gamma$ listed in the theorem, the automorphism groups of all elements of $\mathscr{M}(\Gamma)$ contain the corresponding group $H_{0}$.

1. All Riemann surfaces of genus 2 are hyperelliptic. 
2. All Riemann surfaces of genus 1 with distinguished points $p_{1} \neq p_{2}$ have the automorphism $\alpha(p)=p_{1}+p_{2}-p$.

3. All Riemann surfaces of genus 1 with distinguished point $p_{1}$ have the automorphism $\alpha(p)=2 p_{1}-p$.

4 , 5. The automorphism group of $\mathbb{P}^{1}$ with any four distinct distinguished points contains $\mathbb{Z}_{2} \times \mathbb{Z}_{2}$.

6, 7, 8. $\operatorname{Aut}\left(\mathbb{P}^{1} \sim\{0,1, \infty\}\right)=S_{3}$.

Thus the theorem gives the conditions for some or all of these automorphisms to lift to a regular cover branched over the distinguished points.

This also shows that we can say more in cases 4 and 6 when $k=l$. Suppose $\Gamma$ has signature $[0 ; k, k, k, k]$, corresponding to $\mathbb{P}^{1}$ with four distinguished points $p_{1}, p_{2}, p_{3}, \dot{p}_{4}$. Every orientation preserving automorphism of $\Gamma$ sends $z_{i}$ to a conjugate of some $z_{j}$ and $\operatorname{Mod}(\Gamma)$ maps onto $S_{4}$. The automorphism group noted above permutes $p_{1}, p_{2}, p_{3}, p_{4}$ as the normal $\mathbb{Z}_{2} \times \mathbb{Z}_{2}$ in $S_{4}$. There are three elements of order 2 and any one could lift to the cover of $\mathbb{P}^{1}$ determined by $\psi$. Thus case 4 says that, up to composing $\psi$ with one of three coset representatives of $N\left(\Gamma_{0}\right)$ in Aut $^{+}(\Gamma), l_{Z_{1}}$ induces an automorphism of $G$, or, equivalently, in the notation of case 5 , one of $l_{Z_{1}}, l_{Z_{2}}$, or $l_{Z_{3}}$ induces an automorphism of $G$. Similar remarks hold for case 6 with $k=l$, since $\operatorname{Mod}(\Gamma)=S_{3}$, which has three elements of order 2 .

Remark. Note that the dimension of $\mathscr{M}_{g}^{[G]}$ is 3 in case 1, 2 in case 2, 1 in cases $3,4,5$, and 0 in cases 6, 7, 8 .

Remark. Again suppose $\psi$ and $i$ are as in (1). The above considerations could be applied to any finite subgroup $H_{0}$ of $\operatorname{Mod}(\Gamma)$ to determine whether the automorphisms determined by $H_{0}$ of surfaces uniformized by $\Gamma$ lift to the cover determined by $i$. This is equivalent to asking if $H_{0}<\operatorname{Mod}(\Gamma, K)$, that is, is there a subgroup $H$ of $\operatorname{Mod}_{g}$ so that $G \triangleleft H, H / G \simeq H_{0}$, and $\bar{i}\left(T(\Gamma)^{H_{0}}\right)=T_{g}^{H}$ ? Again, one needs a group $\Gamma_{0}$ of Fuchsian type, an inclusion $j: \Gamma \rightarrow \Gamma_{0}$, and an epimorphism $\theta_{0}: \Gamma_{0} \rightarrow H_{0}$ such that $\operatorname{ker} \theta_{0} \simeq j(\Gamma)$ and $\left(j, \theta_{0}\right)$ determines $H_{0}$ as a subgroup of $\operatorname{Mod}(\Gamma)$. Thus the reason that the above results are so easily computable is that in those cases $H_{0}$ is (or is very nearly) a normal subgroup of $\operatorname{Mod}(\Gamma)$.

It is also possible that $G<H$ are finite subgroups of $\operatorname{Mod}_{g}, G$ not normal in $H$, and $\mathscr{M}_{g}^{[G]}=\mathscr{M}_{g}^{[H]}$. Then, as in the theorem, we have Fuchsian groups $\Gamma<\Gamma_{0}<\operatorname{Aut}^{+}\left(K_{g}\right), \Gamma$ not normal in $\Gamma_{0}, K_{g}$ normal in $\Gamma$, and $\Gamma_{0}$ with $\Gamma / K_{g} \simeq G$ and $\Gamma_{0} / K_{g} \simeq H$, and if $j: \Gamma \rightarrow \Gamma_{0}$ is the inclusion, then $\bar{j} T\left(\Gamma_{0}\right)=$ $T(\Gamma)$. Singerman lists all the possibilities in [18]; all such pairs are triangle groups and so $\mathscr{M}_{g}^{[G]}$ is a single point. Thus if $\mathscr{M}_{g}^{[G]}$ is not a single point and is not a case of the above theorem, then the generic element of $\mathscr{M}_{g}^{[G]}$ has automorphism group isomorphic to $G$.

Let $\Lambda$ be the largest normal subgroup of $\Gamma_{0}$ contained in $\Gamma$. Then, if $T\left(\Gamma_{0}\right)=\{[r]\}$, the map $U / r(\Lambda) \rightarrow U / r\left(\Gamma_{0}\right)$ is the Galois closure of the map $U / r(\Gamma) \rightarrow U / r\left(\Gamma_{0}\right)$. Let $H_{0}=\Gamma_{0} / \Lambda, G_{0}=\Gamma / \Lambda$, and $n=\left[\Gamma_{0}: \Gamma\right]=$ $\left[H_{0}: G_{0}\right]$. Singerman gives in [18] the permutation representation, $\theta_{0}: \Gamma_{0} \rightarrow$ $S_{n}$, of $\Gamma_{0}$ on the set of cosets $\Gamma_{0} / \Gamma$. Then $\operatorname{ker} \theta_{0}=\Lambda$ and $\theta_{0}(\Gamma)=\theta_{0}\left(\Gamma_{0}\right) \cap$ $S_{n-1} \simeq G_{0}$. One can easily see that the signatures of $\Gamma$ and $\Gamma_{0}$ (and hence the index $n$ ) determine the cycle structure of the elements $\theta_{0}\left(Z_{1}\right), \theta_{0}\left(Z_{2}\right)$, and 
$\theta_{0}\left(Z_{3}\right)$ of $S_{n}$, and consequently $\theta_{0}$ is uniquely determined up to composition with an inner automorphism of $S_{n}$. On the other hand, in each case given $\Gamma_{0}$ and $H_{0}$, there is a unique epimorphism $\theta_{0}: \Gamma_{0} \rightarrow H_{0}$ up to composition with $\operatorname{Aut}\left(H_{0}\right)$, and then $\Gamma \simeq \theta_{0}^{-1}\left(G_{0}\right)$ and $\Lambda=\operatorname{ker} \theta_{0}$. Finally suppose $H_{0}$ and $\Lambda$ are given and let $\Lambda$ have signature $\left[h ; k_{1}, \ldots, k_{r}\right]$. Then the signature of $\Gamma_{0}$ is determined and in each case there is a unique suitably pointed Riemann surface of genus $h$ whose automorphism group contains (and hence equals) $H_{0}$.

In Table 1 we reproduce Singerman's list and identify $H_{0}, G_{0}$, and $\Lambda$. Again suppose $T\left(\Gamma_{0}\right)=\{[r]\}$ and let $S_{1}=U / r(\Lambda)$. In cases A and C, $S_{1}$ is the Klein-Hurwitz surface of genus 3. In B, $S_{1}$ is Macbeath's surface of genus 7. In $\mathrm{D}, S_{1}$ is the Fermat quartic of genus 3 and hence $H_{1}=\mathbb{Z}_{4} \times \mathbb{Z}_{4} \rtimes S_{3}$ of order 96 , where $S_{3}$ acts as in the nontrivial component of the permutation representation. $G_{1}$ is any cyclic subgroup of order 8 . In $\mathrm{F}, H_{2}=\mathbb{Z}_{3} \times \mathbb{Z}_{3} \times \mathbb{Z}_{3} \rtimes A_{4}$ of order 324, where again $A_{4}$ acts as in the nontrivial component of the permutation representation. $G_{2}$ has order 27 and is generated by any two elements $g_{1}, g_{2}$ of order 9 such that $\left\langle g_{1}\right\rangle \cap\left\langle g_{2}\right\rangle$ has order 3. In G, $S_{1}$ is Bring's surface of genus 4.

Suppose $\Gamma$ is from the table, $\psi: \Gamma \rightarrow G$ is an epimorphism, and $i$ is an isomorphism from $K_{g}$ to $\operatorname{ker} \psi$. Then the pair $(i, \psi)$ allows us to identify $G$ with a subgroup of $\operatorname{Mod}_{g}$. Suppose $S$ is the surface of genus $g$ with this $G$-action. Then there is a group $H<\operatorname{Mod}_{g}$ with $G<H, G$ not normal in $H$, and $\mathscr{M}_{g}^{[G]}=\mathscr{M}_{g}^{[H]}$ if and only if there is an inclusion $j: \Gamma \rightarrow \Gamma_{0}$ for some $\Gamma_{0}$ corresponding to $\Gamma$ in the table and an epimorphism $\psi_{0}: \Gamma_{0} \rightarrow H$ such that $\psi_{0} \circ j=\psi$ and $\operatorname{ker} \psi_{0}=j(i(K))$. We will not go through the details of each case, but make the following remarks.

TABLE 1

\begin{tabular}{|c|c|c|c|c|c|c|}
\hline & $\Gamma$ & $\Gamma_{0}$ & $H_{0}$ & $G_{0}$ & $\Lambda$ & $n$ \\
\hline $\mathrm{A}$ & {$[0 ; 7,7,7]$} & {$[0 ; 2,3,7]$} & $\operatorname{PSL}(2,7)$ & $\mathbb{Z}_{7}$ & {$[3 ;-]$} & 24 \\
\hline $\mathrm{B}$ & {$[0 ; 2,7,7]$} & {$[0 ; 2,3,7]$} & $\operatorname{PSL}\left(2,2^{3}\right)$ & $\operatorname{Aff}\left(1,2^{3}\right)$ & {$[7 ;-]$} & 9 \\
\hline $\mathrm{C}$ & {$[0 ; 3,3,7]$} & {$[0 ; 2,3,7]$} & $\operatorname{PSL}(2,7)$ & $\operatorname{Aff}^{+}(1,7)$ & {$[3 ;-]$} & 8 \\
\hline $\mathrm{D}$ & {$[0 ; 4,8,8]$} & {$[0 ; 2,3,8]$} & $H_{1}$ & $G_{1}$ & {$[3 ;-]$} & 12 \\
\hline $\mathrm{E}$ & {$[0 ; 3,8,8]$} & {$[0 ; 2,3,8]$} & $\operatorname{PGL}\left(2,3^{2}\right)$ & $\operatorname{Aff}\left(1,3^{2}\right)$ & {$[16 ;-]$} & 10 \\
\hline $\mathrm{F}$ & {$[0 ; 9,9,9]$} & {$[0 ; 2,3,9]$} & $H_{2}$ & $G_{2}$ & {$[10 ;-]$} & 12 \\
\hline $\mathrm{G}$ & {$[0 ; 4,4,5]$} & {$[0 ; 2,4,5]$} & $\operatorname{PGL}(2,5)$ & $\operatorname{Aff}(1,5)$ & {$[4 ;-]$} & 6 \\
\hline $\mathrm{H}$ & {$[0 ; \underset{(m \geq 2)}{m, 4 m, 4 m]}$} & {$[0 ; 2,3,4 m]$} & $S_{4}$ & $\mathbb{Z}_{4}$ & {$[0 ; m, m, m, m$} & 6 \\
\hline $\mathrm{I}$ & {$[0 ; m, 2 m, 2 m]$} & {$[0 ; 2,4,2 m]$} & $D_{8}$ & $\mathbb{Z}_{2}$ & {$[0 ; m, m, m]$} & 4 \\
\hline $\mathrm{J}$ & {$[0 ; \underset{(m \geq 3)}{m, 3,3 m]}$} & {$[0 ; 2,3,3 m]$} & $A_{4}$ & $\mathbb{Z}_{3}$ & {$[0 ; m, m, m]$} & 4 \\
\hline $\mathrm{K}$ & {$[0 ; 2, m, 2 m]$} & {$[0 ; 2,3,2 m]$} & $S_{3}$ & $\mathbb{Z}_{2}$ & {$[0 ; m, m, m]$} & 3 \\
\hline
\end{tabular}


If $j$ and $\psi_{0}$ exist, then (a) there is a homomorphism $\pi: G \rightarrow H_{0}$ such that $\pi \circ \psi=\theta_{0} \circ j$, and then $\pi(G)$ is (conjugate to) $G_{0}$. This says that the quotient map $S \rightarrow S / G$ factors through the surface $S_{1}$ mentioned above. If $k: \Lambda \rightarrow \Gamma$ is an isomorphism from $\Lambda$ to $\operatorname{ker}(\pi \circ \psi)$ and $F=\operatorname{ker} \pi$, then let $\psi_{1}=\psi \circ k$ be the epimorphism from $\Lambda$ onto $F$. If conjugation by $\Gamma_{0}$ acts on $\Lambda$ via $j \circ k$, then (b) for every $\gamma_{0} \in \Gamma_{0}$ there is an $\alpha_{\gamma_{0}} \in \operatorname{Aut}(F)$ so that $\psi_{1} \circ l_{\gamma_{0}}=\alpha_{\gamma_{0}} \circ \psi_{1}$. This says that $H_{0}=\operatorname{Aut}\left(S_{1}\right)$ lifts to $S$. Conversely if (a) and (b) hold for a given $j$, then $\psi_{0}$ exists.

Conversely we could also ask if a given finite subgroup $H$ of $\operatorname{Mod}_{g}$ contains a subgroup $G$ such that $\mathscr{M}_{g}^{[G]}=\mathscr{M}_{g}^{[H]}$. Given all the information above we can give an easily computable condition in the next theorem. We continue the above notation and let $G_{0}=\langle 1\rangle$ in the context of the previous theorem. Note that $\theta_{0}$ depends on the particular case of that theorem and not just on $\Gamma_{0}$ and $H_{0}$. In cases 2,4 , and 6 the epimorphism $\theta_{0}$ given above is not the only one having a group of the "type" of $\Gamma$ as kernel. In cases 2 and 4 when $k=2$ and in case 6 when $l=2 k, \Gamma$ is not a characteristic subgroup of $\Gamma_{0}$. In addition, in case 6 when $l$ is even we may switch the roles of $Z_{2}$ and $Z_{3}$.

Theorem. Suppose $\psi_{0}: \Gamma_{0} \rightarrow H$ determines the conjugacy class $\mathscr{G}_{\psi_{0}}$ of finite subgroups of $\operatorname{Mod}_{g}$. There is a conjugacy class $\mathscr{G}$ of finite subgroups of $\operatorname{Mod}_{g}$ such that $\mathscr{G}<\mathscr{G}_{\psi_{0}}, \mathscr{G} \neq \mathscr{G}_{\psi_{0}}$, and $\mathscr{M}_{g}^{\mathscr{G}}=\mathscr{M}_{g}^{\mathscr{G}_{\psi_{0}}}$ iff $\Gamma_{0}$ is from the previous theorem or Table 1 and there is an epimorphism $\pi: H \rightarrow H_{0}$ such that $\pi \circ \psi_{0}=$ $\theta_{0}$, except in the following cases of the theorem:

2. When $k=2, \pi \circ \psi_{0}\left(Z_{i}\right)=-1$ for all but one generator of $\Gamma_{0}$.

4. When $k=2, \pi \circ \psi_{0}\left(Z_{i}\right)=-1$ for any two of $Z_{1}, Z_{2}, Z_{3}$.

6. When $l$ is even, $\pi \circ \psi_{0}\left(Z_{i}\right)=-1$ for $Z_{1}$ and any one of $Z_{2}, Z_{3}$.

In each case $\mathscr{G}=\mathscr{G}_{\psi}$, where $\psi: \Gamma \rightarrow G$ is defined by letting $G=\pi^{-1}\left(G_{0}\right), j$ be an isomorphism of $\Gamma$ with $\psi_{0}^{-1}(G)$, and $\psi=\psi_{0}^{\circ} j$.

We mention one consequence of the above.

Corollary. Suppose $S$ is a Hurwitz surface with Hurwitz group $H$ and further suppose there is no epimorphism from $H$ to either $\operatorname{PSL}(2,7)$ or $\operatorname{PSL}\left(2,2^{3}\right)$. Then no proper subgroup of $H$ "determines" $S$, that is, for every proper subgroup $G$ of $H, \mathscr{M}_{g}^{[G]}$ has positive dimension.

Finally suppose that $\mathscr{G}_{1}$ and $\mathscr{G}_{2}$ are conjugacy classes of finite subgroups of $\operatorname{Mod}_{g}$ such that $\mathscr{M}_{g}^{\mathscr{G}_{1}}=\mathscr{M}_{g}^{\mathscr{G}_{2}}$. Suppose $G_{i} \in \mathscr{G}_{i}$ are chosen so that $T_{g}^{G_{1}}=T_{g}^{G_{2}}$ and neither $G_{1}$ nor $G_{2}$ contains the other. Let $H$ be the subgroup of $\operatorname{Mod}_{g}$ generated by $G_{1}$ and $G_{2}$. Then $T_{g}^{H}=T_{g}^{G_{1}}$ and $H$ is a finite group. Note that it need not be true that $T_{g}^{G_{1} \cap G_{2}}=T_{g}^{G_{1}}$. Let $\Gamma_{0}, \Gamma_{1}, \Gamma_{2}$, and $\Gamma_{3}$ be the subgroups of Aut ${ }^{+}\left(K_{g}\right)$ projecting to $H, G_{1}, G_{2}$, and $G_{1} \cap G_{2}$, resp. Then the pairs $\Gamma_{1}, \Gamma_{0}$ and $\Gamma_{2}, \Gamma_{0}$ must come from the first theorem or from Table 1.

We will consider the case where the dimension of $\mathscr{M}_{g}^{[H]}$ is greater than or equal to 1. Then $G_{1}$ and $G_{2}$ are normal subgroups of $H$. Let $F_{3}=$ $H /\left(G_{1} \cap G_{2}\right)=\Gamma_{0} / \Gamma_{3}$ and $F_{i}=H / G_{i}=\Gamma_{0} / \Gamma_{i}$. Up to switching $\Gamma_{1}$ and $\Gamma_{2}$ there are only four possibilities which we record in Table 2 . This should be compared to the theorem in $\S 2$ of [6]. 
TABLE 2

\begin{tabular}{|c|c|c|c|c|c|c|}
\hline$\Gamma_{0}$ & $\Gamma_{1}$ & $\Gamma_{2}$ & $\Gamma_{3}$ & $F_{1}$ & $F_{2}$ & $F_{3}$ \\
\hline$[0 ; 2,2,2,2,2]$ & {$[1 ; 2,2]$} & {$[1 ; 2,2]$} & {$[2 ;-]$} & $\mathbb{Z}_{2}$ & $\mathbb{Z}_{2}$ & $\mathbb{Z}_{2} \times \mathbb{Z}_{2}$ \\
\hline $\begin{array}{c}{[0 ; 2,2,2,2 k]} \\
(k \geq 2)\end{array}$ & {$[0 ; 2,2 k, 2,2 k]$} & {$[1 ; k]$} & {$[1 ; k, k]$} & $\mathbb{Z}_{2}$ & $\mathbb{Z}_{2}$ & $\mathbb{Z}_{2} \times \mathbb{Z}_{2}$ \\
\hline $\begin{array}{c}{[0 ; \underset{(k \geq 3)}{2,2,2, k]}} \\
{[0 ; \underset{(k \geq 2)}{(2,2,2 k]}}\end{array}$ & {$[0 ; 2 k, 2 k, 2 k, 2 k]$} & {$[1 ; k]$} & {$[1 ; k, k, k, k]$} & $\mathbb{Z}_{2} \times \mathbb{Z}_{2}$ & $\mathbb{Z}_{2}$ & $\mathbb{Z}_{2} \times \mathbb{Z}_{2} \times \mathbb{Z}_{2}$ \\
\hline
\end{tabular}

Notation. We introduce the following notation for the sake of brevity. Given $\Gamma$ with signature $\left[p ; m_{1}, \ldots, m_{t}\right]$ and presentation (2), we specify homomorphisms $\psi: \Gamma \rightarrow G$ by listing the images of the generators and we will write

$$
\psi \sim\left(\psi\left(x_{1}\right), \psi\left(y_{1}\right), \ldots, \psi\left(z_{t}\right)\right) \in G^{2 p+t} .
$$

Suppose $g \geq 2$ and the finite group $G$ are fixed. Let $\left[G_{1}\right], \ldots,\left[G_{m}\right]$ be all the conjugacy classes of finite subgroups of $\operatorname{Mod}_{g}$ isomorphic to $G$. Then

$$
\mathscr{M}_{g}^{G}=\bigcup_{i=1}^{m} \mathscr{M}_{g}^{\left[G_{i}\right]}
$$

is the space of all Riemann surfaces $S$ of genus $g$ having a subgroup of its automorphism group isomorphic to $G$. It is a finite union of subvarieties of $\mathscr{M}_{g}$, but in general the number of components of $\mathscr{M}_{g}^{G}$ is less than $m$. That is, there may be classes $\left[G_{1}\right]$ and $\left[G_{2}\right]$ such that $G_{1} \simeq G_{2} \simeq G$ and $\mathscr{M}_{g}^{\left[G_{1}\right]} \subset \mathscr{M}_{g}^{\left[G_{2}\right]}$.

We consider the case where $G=\left\langle\delta \mid \delta^{n}=1\right\rangle$ is cyclic of order $n$ for some $n \geq 3$. Suppose $\psi: \Gamma \rightarrow G$ determines the family $\mathscr{M}_{g}^{\mathscr{S}_{\psi}}$. We refer to the cases of the first theorem in $\S 2$.

In cases 1 and $2, H \simeq D_{2 n}$, the dihedral group of order $2 n$, and is the automorphism group of the generic element $S$ of $\mathscr{M}_{g}^{\mathscr{G}_{\psi}}$. In case $1, g=n+1$, and in case $2, g=n-n / k+1$, where $1<k \mid n$. Hence, generically, $\operatorname{Aut}(S)$ contains only one subgroup isomorphic to $G$. In case $3, \Gamma$ has no abelian quotient with torsion free kernel.

Suppose in case 4 that $c_{1}=\psi\left(z_{1}\right)$ is a generator of $G$. By composing $\psi$ with an automorphism of $G$ we may assume $c_{1}=\delta$. We also assume that $H$ is abelian, that is, that $\alpha$ is the identity automorphism of $G$. Then $H \simeq \mathbb{Z}_{n} \times \mathbb{Z}_{2}$ and $\psi \sim\left(\delta, \delta^{e}, \delta, \delta^{e}\right)$ for some $e$ such that $1 \leq e \leq n-1$ and $2+2 e \equiv 0$ $(\bmod n)$. Hence $g=n-(n, e)$. If $n$ is odd, then $e=n-1$ and if $n=2 n_{1}$ is even, then there are two solutions $e=n-1$ and $e=n_{1}-1$.

First suppose $e$ is odd. Note that this implies $n=2 n_{1}$ and $g=n-1$. Then $\Gamma_{0}$ has signature $\left[0 ; 2,2,2 n_{1}, 2 n_{1}\right]$ and

$$
\psi_{0} \sim\left(a, a \delta^{-e-1}, \delta, \delta^{e}\right)=\left(a, a \delta_{1}^{-e-1}, a \delta_{1}, a \delta_{1}^{e}\right),
$$

where $\delta_{1}=a \delta$ also has order $n$. Suppose $\pi: H \rightarrow \mathbb{Z}_{2}$ is the epimorphism with kernel generated by $\delta_{1}$. If $\Gamma_{1}=\operatorname{ker}\left(\pi \circ \psi_{0}\right)$, then $i\left(K_{g}\right) \triangleleft \Gamma_{1} \triangleleft \Gamma_{0}$. From 
(4) we see that each generator of $\Gamma_{0}$ is sent to the generator of $\mathbb{Z}_{2}$. Again an easy computation shows that, if

$$
x_{1}=Z_{1} Z_{2}, \quad y_{1}=Z_{3} Z_{2}, \quad z_{1}=Z_{3}^{2}, \quad z_{2}=Z_{4}^{2},
$$

then $x_{1}, y_{1}, z_{1}, z_{2}$ generate $\Gamma_{1}$ with relations $\left[x_{1}, y_{1}\right] z_{1} z_{2}=1=z_{1}^{n_{1}}=z_{2}^{n_{1}}$. Thus $\Gamma_{1}$ has signature $\left[1 ; n_{1}, n_{1}\right]$ and if $\psi_{1}=\psi_{0} \mid \Gamma_{1}: \Gamma_{1} \rightarrow G_{1}=\left\langle\delta_{1}\right\rangle$, then $\psi_{1} \sim\left(\delta_{1}^{-e-1}, \delta_{1}^{e} ; \delta_{1}^{2}, \delta_{1}^{-2}\right)$. Thus $\mathscr{M}_{g}^{\mathscr{S}_{\psi}} \subset \mathscr{M}_{g}^{\mathscr{S}_{\psi_{1}}}$.

Remark. If the Reimann surface $S$ corresponds to an element of $\mathscr{M}_{g}^{\mathscr{G}_{\psi}}$, then the projection from $S$ to $S / G_{1}$, the quotient by the action of $G_{1}$, is a map to a Riemann surface of genus 1 with two branch points. Since

$$
\begin{gathered}
l_{Z_{2}}\left(x_{1}\right)=x_{1}^{-1}, \quad l_{Z_{2}}\left(y_{1}\right)=y_{1}^{-1} z_{1}, \\
l_{Z_{2}}\left(z_{1}\right)=y_{1}^{-1} z_{1} y_{1}, \quad l_{Z_{2}}\left(z_{2}\right)=\left(x_{1} y_{1}\right)^{-1} z_{2}\left(x_{1} y_{1}\right),
\end{gathered}
$$

we see that the automorphism of order two of the quotient surface $S / G_{1}$ which lifts to $S$ to give the automorphism $a$ in $H$ fixes both ramification points, and so is not the automorphism of order 2 possessed by all elements of $\mathscr{M}_{g}^{\mathscr{G}_{\psi_{1}}}$ as in case 2 .

Remark. If $n=4 n_{0}+2$, then only $e=4 n_{0}+1$ is odd and corresponds to $g=n-1$.

Remark. If $n=4 n_{0}$, then $e=4 n_{0}-1$ and $e^{\prime}=2 n_{0}-1$ are both odd and correspond to $g=4 n_{0}-1$. Then by the construction above we get two homomorphisms $\psi$ and $\psi^{\prime}$ from $\Gamma$ to $G$ which determine two homomorphisms $\psi_{1} \sim\left(1, \delta_{1}^{-1} ; \delta_{1}^{2}, \delta_{1}^{-2}\right)$ and $\psi_{1}^{\prime} \sim\left(\delta_{1}^{2 n_{0}}, \delta_{1}^{2 n_{0}-1} ; \delta_{1}^{2}, \delta_{1}^{-2}\right)$ from $\Gamma_{1}$ with signature $\left[1 ; 2 n_{0}, 2 n_{0}\right]$ to $G_{1}$. Without supplying details [1,7], we claim that there is a $\beta \in \operatorname{Aut}^{+}\left(\Gamma_{1}\right)$ so that $\psi_{1} \circ \beta=\psi_{1}^{\prime}$. Since $\psi$ and $\psi^{\prime}$ are not equivalent, we have three distinct conjugacy classes $\mathscr{G}_{\psi}, \mathscr{G}_{\psi^{\prime}}$, and $\mathscr{G}_{\psi_{1}}$ of cyclic groups of order $4 n_{0}$ such that $\mathscr{M}_{g}^{\mathscr{S}_{\psi}} \cup \mathscr{M}_{g}^{\mathscr{G}_{\psi^{\prime}}} \subset \mathscr{M}_{g}^{\mathscr{G}_{\psi_{1}}}$.

Now suppose $n$ and $e$ are even. Then $n=4 n_{0}+2, e=2 n_{0}$, and $g=$ $n-2=4 n_{0}$. Then $\Gamma_{0}$ has signature $\left[0 ; 2,2,4 n_{0}+2,2 n_{0}+1\right]$ and

$$
\psi_{0} \sim\left(a, a \delta^{2 n_{0}+1}, \delta, \delta^{2 n_{0}}\right)=\left(a, \delta_{1}^{2 n_{0}+1}, a \delta_{1}, \delta_{1}^{2 n_{0}}\right),
$$

where $\delta_{1}=a \delta$ also has order $n$. Suppose $\pi: H \rightarrow \mathbb{Z}_{2}$ is the epimorphism with kernel generated by $\delta_{1}$. If $\Gamma_{1}=\operatorname{ker}\left(\pi \circ \psi_{0}\right)$, then $i\left(K_{g}\right) \triangleleft \Gamma_{1} \triangleleft \Gamma_{0}$ and $\pi \circ \psi_{0}$ sends the generators $Z_{1}$ and $Z_{3}$ of $\Gamma_{0}$ to the generator of $\mathbb{Z}_{2}$, and $Z_{2}$ and $Z_{4}$ to the identity. If

$$
z_{1}=Z_{1} Z_{2} Z_{1}, \quad z_{2}=Z_{2}, \quad z_{3}=Z_{3}^{2}, \quad z_{4}=Z_{3}^{-1} Z_{4} Z_{3}, \quad z_{5}=Z_{4},
$$

then $z_{1}, \ldots, z_{5}$ generate $\Gamma_{1}$ and it is easy to see that $\Gamma_{1}$ has signature $\left[0 ; 2,2,2 n_{0}+1,2 n_{0}+1,2 n_{0}+1\right]$. If $\psi_{1}=\left.\psi_{0}\right|_{\Gamma_{1}}: \Gamma_{1} \rightarrow G_{1}=\left\langle\delta_{1}\right\rangle$, then

$$
\psi_{1} \sim\left(\delta_{1}^{2 n_{0}+1}, \delta_{1}^{2 n_{0}+1}, \delta_{1}^{2}, \delta_{1}^{2 n_{0}}, \delta_{1}^{2 n_{0}}\right)
$$

and we have $\mathscr{M}_{g}^{\mathscr{G}_{\psi}} \subset \mathscr{M}_{g}^{\mathscr{S}_{\psi_{1}}}$.

Remark. If $n$ is odd, then $H \simeq \mathbb{Z}_{2 n}$ and does not contain a second subgroup isomorphic to $\mathbb{Z}_{n}$. 
Remark. If $e=n-1$, then $\psi$ satisfies the hypotheses of case 5 with $\beta(\delta)=\delta^{-1}$ and the automorphism group of the generic element of $\mathscr{M}_{g}^{\mathscr{S}_{\psi}}$ is isomorphic to $H=D_{2 n} \times \mathbb{Z}_{2}$. If $\Gamma_{0}$ has signature $[0 ; 2,2,2, n]$, then $\psi_{0}: \Gamma_{0} \rightarrow H$ is given by $\psi_{0} \sim\left(b, b \delta^{-1} a, a, \delta\right)$. From the form of $\psi_{0}$ we can see that $a$ has $2 n$ fixed points and so must be the hyperelliptic involution. Thus all elements of $\mathscr{M}_{g}^{\mathscr{S}_{\psi}}$ are hyperelliptic. If in addition $n=2 n_{1}$, then $\psi_{0}$ satisfies case 3 . If $\Gamma_{2}$ has signature $\left[1 ; n_{1}\right]$ and $G_{2}=\langle a \delta, a b\rangle \simeq D_{2 n}$, we get $\psi_{2}: \Gamma_{2} \rightarrow G_{2}$ defined by $\psi_{2} \sim\left(\delta^{-1} a, a b ; \delta^{2}\right)$ and $\mathscr{M}_{g}^{\mathscr{S}_{\psi}}=\mathscr{M}_{g}^{\mathscr{S}_{\psi_{2}}}$, where $g=n-1$. Thus $\psi$ and $\psi_{2}$ give us an example of the fourth case of Table 2. Note that $G \cap G_{2}=\left\langle\delta^{2}\right\rangle \simeq \mathbb{Z}_{n_{1}}$ and $G$ is not conjugate to a subgroup of $G_{2}$ in $\operatorname{Mod}_{g}$.

If $n=4 n_{0}$ and $e=2 n_{0}-1$, then $e^{2} \equiv 1(\bmod n)$ and again $\psi$ satisfies the hypotheses of case 5 with $\beta(\delta)=\delta^{e}$. It can be seen from this that the general element of this $\mathscr{M}_{g}^{\mathscr{S}_{\psi}}$ is not hyperelliptic. Note that for $n_{0}=e=1$, this family of genus 3 Riemann surfaces contains the Fermat quartic.

In [16] the Jacobi varieties of the hyperelliptic examples above were studied. Some other examples of genus 3 with automorphism group containing $S_{4}$ were also studied. Suppose $\Gamma$ has signature $[0 ; 4,4,3]$ and $\psi: \Gamma \rightarrow S_{4}$ is the epimorphism defined by $\psi \sim((1243),(1234),(123))$. Then $\alpha=l_{(34)}$ satisfies the hypothesis of case 6 . Let $a_{0}=a(34)$. Then $a_{0}^{2}=1$ and $a_{0}$ commutes with $G$ and so $H=G \times\left\langle a_{0}\right\rangle$. From the proof of case 6 , we have $\Gamma_{0}$ has signature $[0 ; 2,4,6]$ and $\psi_{0}: \Gamma_{0} \rightarrow H$ is determined by

$$
\psi_{0} \sim(a,(1234), a(1342))=\left(a_{0}(34),(1234), a_{0}(132)\right) .
$$

There is a second subgroup of $H$ isomorphic to $S_{4}$; we identify $G$ with this subgroup by composing all elements of $S_{4}-A_{4}$ with $a_{0}$. Then $\psi_{0}$ becomes

$$
\psi_{0} \sim\left((34), a_{0}(1234), a_{0}(132)\right) .
$$

Now suppose $\pi: H \rightarrow \mathbb{Z}_{2}$ is the epimorphism whose kernel is the new $G$. If $\Gamma_{1}=\operatorname{ker}\left(\pi \circ \psi_{0}\right)$, then $i\left(K_{3}\right) \triangleleft \Gamma_{1} \triangleleft \Gamma_{0}$ and $\pi \circ \psi_{0}$ sends the generators $Z_{2}$ and $Z_{3}$ of $\Gamma_{0}$ to the generator of $\mathbb{Z}_{2}$, and sends $Z_{1}$ to the identity. If

$$
z_{1}=Z_{1}, \quad z_{2}=Z_{2} Z_{1} Z_{2}^{-1}, \quad z_{3}=Z_{2}^{2}, \quad z_{4}=Z_{3}^{2},
$$

then $z_{1}, z_{2}, z_{3}, z_{4}$ generate $\Gamma_{1}$, and $\Gamma_{1}$ has signature $[0 ; 2,2,2,3]$. If $\psi_{1}=\left.\psi_{0}\right|_{\Gamma_{1}}: \Gamma_{1} \rightarrow G$, then

$$
\psi_{1} \sim((34),(23),(13)(24),(123)),
$$

which is equivalent to the example in [16]. Hence $\mathscr{M}_{3}^{\mathscr{S}_{\psi}} \subset \mathscr{M}_{3}^{\mathscr{S}_{\psi_{1}}}=\mathscr{M}_{3}^{G}$.

Remark. Suppose $\Gamma_{2}$ has signature $[0 ; 2,2,2,2,2,2]$ and $\psi_{2}: \Gamma_{2} \rightarrow \mathbb{Z}_{2} \times \mathbb{Z}_{2}$ is defined by $\psi_{2} \sim\left(\varepsilon_{1}, \varepsilon_{1}, \varepsilon_{2}, \varepsilon_{2}, \varepsilon_{1} \varepsilon_{2}, \varepsilon_{1} \varepsilon_{2}\right)$. Then $\operatorname{ker} \psi_{2} \simeq K_{3}$ and $\psi_{2}$ determines a conjugacy class $\mathscr{G}_{\psi_{2}}$ in $\operatorname{Mod}_{3}$. Using $\psi_{1}$ above we have both $\mathscr{G}_{\psi_{2}}<\mathscr{G}_{\psi_{1}}$ (nonnormally) and $\mathscr{G}_{\psi_{2}} \triangleleft \mathscr{G}_{\psi_{1}}$. This is true since all the induced actions from $\psi_{1}$ of subgroups $\mathbb{Z}_{2} \times \mathbb{Z}_{2}$ of $S_{4}$ are topologically equivalent to $\psi_{2}$.

Since there are unique $S_{4}$ actions in genera 4,5 , and 6 , the next example with $G=S_{4}$ occurs when $g=7$. Here $\Gamma$ has signature $[0 ; 2,4,2,4]$ and $\psi: \Gamma \rightarrow S_{4}$ is defined by $\psi \sim((13),(1432),(14),(1342))$. Then $\alpha=l_{(34)}$ satisfies the hypothesis of case 4 . Again letting $a_{0}=a(34)$ we have $a_{0}^{2}=1$ and 
$H=G \times\left\langle a_{0}\right\rangle ; \Gamma_{0}$ has signature $[0 ; 2,2,2,4]$ and $\psi_{0}: \Gamma_{0} \rightarrow H$ is defined by $\psi_{0} \sim(a, a(12)(34),(13),(1432))=\left(a_{0}(34), a_{0}(12),(13),(1432)\right)$. Again we identify $G$ with the other subgroup of $H$ isomorphic to $S_{4}$ by composing all elements of $S_{4}-A_{4}$ with $a_{0}$. Then $\psi_{0}$ becomes

$$
\psi_{0} \sim\left((34),(12), a_{0}(13), a_{0}(1432)\right) .
$$

Again suppose $\pi: H \rightarrow \mathbb{Z}_{2}$ is the epimorphism with the new $G$ as kernel and $\Gamma_{1}=\operatorname{ker}\left(\pi \circ \psi_{0}\right)$. Then $\pi \circ \psi_{0}$ sends the generators $Z_{1}$ and $Z_{2}$ of $\Gamma_{0}$ to the identity of $\mathbb{Z}_{2}$, and sends $Z_{3}$ and $Z_{4}$ to the generator of $\mathbb{Z}_{2}$. If

$$
z_{1}=Z_{1}, \quad z_{2}=Z_{2}, \quad z_{3}=Z_{3} Z_{1} Z_{3}, \quad z_{4}=Z_{3} Z_{2} Z_{3}, \quad z_{5}=Z_{4}^{2},
$$

then $z_{1}, \ldots, z_{5}$ generate $\Gamma_{1}$, and $\Gamma_{1}$ has signature $[0 ; 2,2,2,2,2]$. If $\psi_{1}=$ $\left.\psi_{0}\right|_{\Gamma_{1}}: \Gamma_{1} \rightarrow G$, then $\psi_{1} \sim((34),(12),(14),(23),(13)(24))$, and $\mathscr{M}_{7}^{\mathscr{G}_{\psi}} \subset$ $\mathscr{M}_{7}^{\mathscr{S}_{\psi_{1}}}=\mathscr{M}_{7}^{G}$.

We have seen that the action of $G$ on a collection of Riemann surfaces of genus $g$ is determined by an epimorphism $\psi: \Gamma \rightarrow G$ whose kernel is isomorphic to $K_{g}$. In this section we will study some topological and analytic invariants associated to an action of $G$. In particular, we will examine to what extent these invariants determine the (equivalence class of ) $\psi$ or can distinguish between two such.

Suppose $S$ is a Riemann surface of genus $g \geq 2$ and $G<\operatorname{Aut}(S)$. Suppose the action of $G$ is determined by $\psi$ and $i$ as in (1) and $S=S_{r}$, where $r=\rho \circ i$ and $[\rho] \in T(\Gamma)$. The action of $\Gamma$ on $K$ via conjugation yields an action of $G$ on $K / K^{\prime} \simeq H_{1}(S, \mathbb{Z})$, where $K^{\prime}$ is the commutator subgroup of $K$. This action preserves the intersection pairing. Choosing a canonical basis [4] in $H_{1}(M, \mathbb{Z})$, we have a representation $R: G \rightarrow \operatorname{Sp}(2 g, \mathbb{Z})$, determined up to conjugation in $\operatorname{Sp}(2 g, \mathbb{Z})$. Let $\chi$ denote the character of $R$.

Let $H^{0}\left(S, \Omega^{q}\right)$ denote the space of holomorphic $q$-differentials on $S$, which we can identify with the space of holomorphic $q$-differentials on $U$ invariant with respect to the (pull-back) action of $r(K)$. Then $\rho(\Gamma)$ preserves this space and induces a (right) action of $G$. The character $\chi_{q}$ of this representation of $G$ is independent of the choice of $\rho$ and of the choice of $[\rho]$ in $T(\Gamma)$.

Remark. Note that $\chi=\chi_{1}+\bar{\chi}_{1}$.

Suppose $p \in S, a \in G, a \neq 1$, and $a(p)=p$. Then there is a local coordinate $z$ centered at $p$ and an integer $u, 1 \leq u<o(a)$, where $o(a)$ denotes the order of $a$, so that $u$ is relatively prime to $o(a)$ and $z \circ a=e^{\frac{2 \pi i u}{(a)}} z$. That is, the transformation $a$ rotates a disk neighborhood around $p$ by the angle $\frac{2 \pi u}{o(a)}$. The integer $u$ is called the rotation number of $a$ at $p$. For $a \in G-\{1\}$ and $u \in \mathbb{Z}$, let $\lambda_{a}(u)$ be the number of points $p$ in $S$ at which $a$ has rotation number congruent to $u$ modulo $o(a)$. Note that $\lambda_{a}(u)=0$ if $(u, o(a)) \neq 1$. The collection $\left\{\lambda_{a} \mid a \in G, a \neq 1\right\}$ is called the rotation data of the action of $G$ on $S$. It can be calculated directly from $\psi$.

Remark. For all $a, b \in G$ and $u, k \in \mathbb{Z}$ with $a \neq 1$, we have $\lambda_{a}=\lambda_{b a b^{-1}}$. If $(k, o(a))=1$, then $\lambda_{a^{k}}(k u)=\lambda_{a}(u)$, and if $k \mid o(a)$, so that $o(a)=k o\left(a^{k}\right)$, then

$$
\lambda_{a^{k}}(u) \geq \lambda_{a}(u)+\lambda_{a}\left(u+o\left(a^{k}\right)\right)+\cdots+\lambda_{a}\left(u+(k-1) o\left(a^{k}\right)\right) .
$$


Remark. If $\psi$ is replaced by $\beta^{-1} \circ \psi$ for some $\beta$ in $\operatorname{Aut}(G)$, then $\chi, \chi_{q}$ and $\lambda_{a}$ are replaced by $\chi \circ \beta, \chi_{q} \circ \beta$, and $\lambda_{\beta(a)}$ respectively.

Eichler's trace formula $[2,4,11]$ states that, for $1 \leq q$ and $a \in G, a \neq 1$,

$$
\chi_{q}(a)=\sum_{u=1}^{o(a)-1} \lambda_{a}(u) \frac{\zeta^{q u}}{1-\zeta^{u}}+\delta_{q, 1}
$$

where $\zeta=e^{\frac{2 \pi i}{o(a)}}$. Since $\chi_{q}(1)=(2 q-1)(g-1)+\delta_{q, 1}$, this shows that the rotation data determines the sequence of characters $\chi_{q}$. We will see that the converse of this statement is also true.

For $F<G$, let $\chi_{F}=\left(1_{F}\right)^{G}$ be the character of the permutation representation of $G$ on the set of cosets $G / F$. In particular, $\chi_{\langle 1\rangle}$ is the character of the regular representation of $G$ and $\chi_{G}$ is the trivial character of $G$. The following are easy to verify. For $b \in G$, let $C(b)$ denote the conjugacy class of $b$ in $G$ and let $\operatorname{Cent}(b)$ be the centralizer of $b$.

(a) $\chi_{F}(b)=|\operatorname{Cent}(b)||C(b) \cap F| /|F|$ and so $\chi_{F}(b)=0$ if and only if no conjugate of $\langle b\rangle$ is contained in $F$.

(b) $\chi_{b F b^{-1}}=\chi_{F}$.

(c) If $(k, o(b))=1$, then $\chi_{F}\left(b^{k}\right)=\chi_{F}(b)$.

(d) Suppose $\langle 1\rangle,\left\langle a_{1}\right\rangle, \ldots,\left\langle a_{s}\right\rangle$ is a list of representatives from the set of conjugacy classes of cyclic subgroups of $G$. Then $\chi_{\langle 1\rangle}, \ldots, \chi_{\left\langle a_{s}\right\rangle}$ form a basis for the space of class functions on $G$ with property (c).

Proposition. The character $\chi$ determines the signature of $\Gamma$.

Proof. Suppose the action of $G$ is determined by the epimorphism $\psi: \Gamma \rightarrow G$ as in (1), where $\Gamma$ has signature $\left[p ; m_{1}, \ldots, m_{t}\right]$ and presentation (2). Let $c_{i}=\psi\left(z_{i}\right)$ for $i=1, \ldots, t$. Then we have [2]

$$
\chi=2 \chi_{G}+(2 p-2+t) \chi_{\langle 1\rangle}-\sum_{i=1}^{t} \chi_{\left\langle c_{i}\right\rangle} .
$$

First note that $p$ is determined, since $2 p=\left\langle\chi, \chi_{G}\right\rangle$. For $l=1, \ldots, s$, let $n_{l}$ be the number of the $c_{i}$ so that $\left\langle c_{i}\right\rangle$ is conjugate to $\left\langle a_{l}\right\rangle$. Then (6) can be rewritten as

$$
\chi=2 \chi_{G}+(2 p-2+t) \chi_{\langle 1\rangle}-\sum_{l=1}^{s} n_{l} \chi_{\left\langle a_{l}\right\rangle} .
$$

Suppose no conjugate of $\left\langle a_{r}\right\rangle$ is contained in any other of the $\left\langle a_{l}\right\rangle$. Then $\chi_{\left\langle a_{l}\right\rangle}\left(a_{r}\right)=0$ if and only if $l \neq r$. Evaluating (7) at $a_{r}$, we obtain

$$
\chi\left(a_{r}\right)=2-n_{r} \frac{\left|N\left(\left\langle a_{r}\right\rangle\right)\right|}{o\left(a_{r}\right)},
$$

which determines $n_{r}$. Suppose that $n_{l}$ is known for all $\left\langle a_{l}\right\rangle$ which contain a conjugate of $\left\langle a_{r}\right\rangle$. Then evaluating (7) at $a_{r}$ yields an equation involving $n_{r}$ and only known $n_{l}$ 's. In this way the $n_{l}$ can be found. Thus the signature of $\Gamma$ is $\left[p ; o\left(a_{1}\right)^{n_{1}}, \ldots, o\left(a_{s}\right)^{n_{s}}\right]$, where $m^{n}$ means that $m$ is repeated $n$ times.

Remark. The proof of the above proposition could be based on the monodromy description of covers of Riemann surfaces (see, for example, Lemma 1.2 of 
[16]). We have the following expressions for the number of fixed points of $a_{l}$ :

$$
2-\chi\left(a_{l}\right)=\sum_{u=1}^{o\left(a_{l}\right)-1} \lambda_{a_{l}}(u)=\sum_{\substack{k=1 \\ C\left(a_{l}\right) \cap\left\langle a_{k}\right\rangle \neq \varnothing}}^{s} n_{k} \frac{\left|N\left(\left\langle a_{k}\right\rangle\right)\right|}{o\left(a_{k}\right)}
$$

Let $L=1$ if $t=0$, and $L=\operatorname{lcm}\left\{m_{1}, \ldots, m_{t}\right\}$ otherwise.

Proposition. For $q \geq 1$ we have

$$
\chi_{q+L}=\chi_{q}+\frac{2 L(g-1)}{|G|} \chi_{\langle 1\rangle}-\delta_{q, 1} \chi_{G}
$$

In particular, if $t=0$ we have

$$
\chi_{q}=(2 q-1)(p-1) \chi_{\langle 1\rangle}+\delta_{q, 1} \chi_{G} .
$$

Proof. Since $o\left(c_{i}^{k}\right)$ divides $L$ for any $k$, the Eichler trace formula (5) implies that for any $a \in G, a \neq 1$,

$$
\chi_{q+L}(a)=\chi_{q}(a)-\delta_{q, 1}
$$

The result follows easily from this. Note that the Riemann-Hurwitz relation implies that

$$
\frac{2 L(g-1)}{|G|}=L\left(2 p-2+\sum_{i=1}^{t}\left(1-\frac{1}{o\left(c_{i}\right)}\right)\right)
$$

is a positive integer.

Proposition. The sequence of characters $\chi_{q}$ determines the rotation data $\lambda$.

Proof. This follows directly from the Eichler trace formula. Fix $a \in G, a \neq 1$. Equation (5) with $q=1, \ldots, \phi(o(a))$ gives a system of linear equations in the unknowns $\lambda_{a}(u) \frac{\zeta^{u}}{1-\zeta^{u}},(u, o(a))=1$, whose coefficient matrix is Vandermonde.

Combining the above results we see that knowing the sequence of characters $\chi_{q}$ is equivalent to knowing the signature of $\Gamma$ and the rotation data $\lambda$. Note that I. Kuribayashi proves the above result in [11], where he obtains the best estimate on the number of $\chi_{q}$ needed to determine $\lambda$.

We now examine some applications of the above to the questions raised at the beginning of this section. We will consider fixed point free actions of $G$ on a Riemann surface $S$ of genus $g$. Then the rotation data $\lambda$ is identically zero and all the characters $\chi_{q}$ are determined by $G$ and $g$. Let $p=\frac{g-1}{|G|}+1$ be the genus of $S / G$ and $\pi: S \rightarrow S / G$ the quotient map.

Suppose first that $G$ is abelian. Then the cover $\pi$ is determined by an injection $j$ of $\widehat{G}$, the character group of $G$, into $H_{1}(S / G, \mathbb{R}) / H_{1}(S / G, \mathbb{Z})$, the real form of the Jacobi variety of $S / G$ (see, for example, [15, 16]). Let $\mathscr{L}$ be the sublattice of $H_{1}(S / G, \mathbb{R})$ such that $j(\widehat{G})=\mathscr{L} / H_{1}(S / G, \mathbb{Z})$. The map $\pi$ induces an injection $\tilde{\pi}: H_{1}(S / G, \mathbb{R}) \rightarrow H_{1}(S, \mathbb{R})$. The image of $\tilde{\pi}$ is $H_{1}(S, \mathbb{R})^{R(G)}$ and $\tilde{\pi}(\mathscr{L})=H_{1}(S, \mathbb{Z})^{R(G)}$. Furthermore, for all $\gamma_{1}$ and $\gamma_{2}$ in $\mathscr{L}$, we have

$$
\left\langle\tilde{\pi}\left(\gamma_{1}\right), \tilde{\pi}\left(\gamma_{2}\right)\right\rangle_{S}=|G|\left\langle\gamma_{1}, \gamma_{2}\right\rangle_{S / G},
$$

where $\langle,\rangle_{S}$ and $\langle,\rangle_{S / G}$ denote the intersection pairings on $S$ and $S / G$ respectively. Then $\tilde{\pi}$ covers the induced map $\tilde{\pi}: J(S / G) \rightarrow J(S)$ between the 
Jacobi varieties of $S / G$ and $S$, the kernel of $\tilde{\pi}$ is $j(\widehat{G})=\mathscr{L} / H_{1}(S / G, \mathbb{Z})$, and $\mathscr{L}^{\perp} / \mathscr{L}$ is the kernel of the polarization induced on $\tilde{\pi}(J(S / G))$ by $J(S)$, where

$$
\mathscr{L}^{\perp}=\left\{\gamma \in H_{1}(S / G, \mathbb{R})|| G \mid\langle\gamma, \delta\rangle_{S / G} \in \mathbb{Z} \text { for all } \delta \in \mathscr{L}\right\} .
$$

Thus the group $\mathscr{L}^{\perp} / \mathscr{L}$ is an invariant of the fixed point free action of $G$ on $S$ and by the above can be calculated from $R$.

For example, suppose $G=\mathbb{Z}_{n} \times \mathbb{Z}_{n}=\left\langle\varepsilon_{1}\right\rangle \times\left\langle\varepsilon_{2}\right\rangle$ and $\Gamma$ has signature $[p ;-]$ and presentation

$$
\left\langle X_{1}, Y_{1}, \ldots, X_{p}, Y_{p} \mid\left[X_{1}, Y_{1}\right] \cdots\left[X_{p}, Y_{p}\right]=1\right\rangle .
$$

Then using automorphisms of $\Gamma$ as in $[1,7]$ it is easy to show that any epimorphism $\psi: \Gamma \rightarrow G$ is equivalent to

$$
\psi_{k} \sim\left(\varepsilon_{1}, \varepsilon_{2}^{k}, \varepsilon_{2}, 1, \ldots, 1\right)
$$

for some positive $k, k \mid n$. The problem now is to show that the $\psi_{k}$ are inequivalent. But an easy calculation shows that for $\psi_{k}$,

$$
\mathscr{L}^{\perp} / \mathscr{L} \simeq\left(\mathbb{Z}_{k}\right)^{2} \times\left(\mathbb{Z}_{n^{2} / k}\right)^{2} \times\left(\mathbb{Z}_{n^{2}}\right)^{2 p-4} .
$$

Thus we can say that the representation $R$ of $G$ distinguishes the fixed point free actions of $G$ on $S$.

If $S / G$ has nontrivial automorphisms, then these may or may not lift to $S$ for different $G$-actions. For example, suppose $S / G$ is hyperelliptic. It is known that the hyperelliptic involution does not lift to every Galois cover. If $G$ has fixed points, then a necessary condition is that the hyperelliptic involution preserves the image of the set of branch points in $S / G$. However, if $G$ is abelian and acts without fixed points, then it always lifts [15]. We will give some examples to show that this is not the case if $G$ is not abelian.

Suppose $\Gamma$ has signature $[p ;-]$ and $G=\operatorname{Aff}^{+}(1,7)$. Then there is one equivalence class of epimorphisms $\psi: \Gamma \rightarrow G$. Since $G$ is solvable, one can see that a lift of the hyperelliptic involution must conjugate an element of order three to its square times an element of order seven. But $G$ has no such automorphism. Thus the hyperelliptic involution cannot lift to any unramified $G$ cover of a surface of genus $p$. The action of $G$ on $\mathbb{F}_{7}$ yields an embedding of $G$ into $S_{7}$. Let $p=2$ and define two homomorphisms $\psi_{1}$ and $\psi_{2}$ from $\Gamma$ to $S_{7}$ by

$$
\begin{gathered}
\psi_{1} \sim((12),(1234567),(1234567),(12)), \\
\psi_{2} \sim((142)(356),(1234567),(147)(26)(35),(1234)) .
\end{gathered}
$$

It is clear that $\psi_{1}$ is an epimorphism. Let $a, b, c, d$ denote the four entries in $\psi_{2}$. The subgroup of $S_{7}$ generated by $a, b, c$, and $d$ is not contained in $A_{7}$. On the other hand $d^{3} c^{2} d=(127)$ and $d^{3} c^{4} d b=(34567)$ and so this subgroup contains $A_{7}$. Thus $\psi_{2}$ is also an epimorphism. It is clear that $\psi_{1}$ satisfies case 1 of the theorem of $\S 2$. But $a$ and $b$ generate a copy of $\operatorname{Aff}^{+}(1,7)$ in $S_{7}$ and so $\psi_{2}$ cannot satisfy case 1 . Thus $\psi_{1}$ and $\psi_{2}$ determine inequivalent unramified $S_{7}$ covers of surfaces of genus 2 .

We now examine the case of fixed point free actions by $D_{2 n}$, the dihedral group of order $2 n$. Suppose $\Gamma$ has signature $[p ;-]$ and presentation (9) and 
$D_{2 n}=\left\langle a, b \mid a^{n}=b^{2}=a b a b=1\right\rangle$. Using the results in [1,7], we can show that any epimorphism $\psi: \Gamma \rightarrow D_{2 n}$ is equivalent to either

$$
\psi_{1} \sim(b, 1, a, 1, \ldots, 1)
$$

or, if $n=2 n_{0}$,

$$
\psi_{2} \sim\left(b, a^{n_{0}}, a, 1, \ldots, 1\right) .
$$

Again the problem is to show that these are inequivalent when $n=2 n_{0}$. Since they determine fixed point free actions of $D_{2 n}$ on surfaces of genus $g=$ $2 n(p-1)+1$, all the characters $\chi_{q}$ agree. We will examine the (symplectic equivalence classes of the) representations $R_{1}$ and $R_{2}$. For simplicity, we assume now that $p=2$.

For $\psi_{1}$ and $\psi_{2}$ we will give canonical bases $\left\{x_{1}, \ldots, x_{2 n+1}, y_{1}, \ldots, y_{2 n+1}\right\}$ of $H_{1}(S, \mathbb{Z})$ and determine the corresponding actions of $D_{2 n}$. Recall that a canonical basis is one with the property

$$
\left\langle x_{i}, x_{j}\right\rangle_{S}=\left\langle y_{i}, y_{j}\right\rangle_{S}=0, \quad\left\langle x_{i}, y_{j}\right\rangle_{S}=\delta_{i j}, \quad 1 \leq i, j \leq 2 n+1 .
$$

We will use the same letter to denote a curve, its homotopy class, or its homology class. We will write the operation in homology as addition, while still writing concatenation of curves multiplicatively, read from left to right.

Suppose $\pi_{1}: S \rightarrow S / D_{2 n}$ is the quotient map for the action of $D_{2 n}$ on $S$ determined by $\psi_{1}$. Let $p_{0} \in S / D_{2 n}$ and label the points of $\pi_{1}^{-1}\left(p_{0}\right)$ with the elements of $D_{2 n}$ so that for $c, d \in D_{2 n}, c \cdot p_{d}=p_{c d}$. Suppose $X \in \Gamma \simeq$ $\pi_{1}\left(S / D_{2 n}, p_{0}\right)$ and $c \in D_{2 n}$. Let $X(c)$ denote the lift of $X$ to $S$ starting at $p_{c}$. Then the other endpoint of $X(c)$ is $p_{c \psi_{1}(X)}$. Also, if $d \in D_{2 n}$, then $d \cdot X(c)=X(d c)$. For example, since $\psi_{1}\left(X_{1}\right)=b, \quad X_{1}(1) X_{1}(b)$ is a closed curve in $S$. Now define

$$
\begin{aligned}
x_{1} & =X_{1}(1) X_{1}(b), & y_{1} & =Y_{1}(b)-Y_{2}(1), \\
x_{k} & =a^{k-1} \cdot x_{1}, & y_{k} & =a^{k-1} \cdot y_{1}, 2 \leq k \leq n, \\
x_{n+1} & =-X_{1}(1) X_{2}(b) X_{1}(b a) X_{2}\left(a^{n-1}\right), & y_{n+1} & =-Y_{2}(1), \\
x_{n+k} & =a^{k-1} \cdot x_{n+1}, & y_{n+k} & =a^{k-1} \cdot y_{n+1}, 2 \leq k \leq n, \\
x_{2 n+1} & =X_{2}(b) X_{2}(b a) \cdots X_{2}\left(b a^{n-1}\right), & y_{2 n+1} & =Y_{2}(b a)-Y_{2}(1) .
\end{aligned}
$$

Since the closed curves which appear on this list either share a segment or intersect only over $p_{0}$, it is not hard to show that the homology classes have the right intersections.

We identify $D_{2 n}$ with a subgroup of $S_{n}$ by setting $a=(12 \cdots n)^{-1}$ and $b=(2 n)(3 n-1) \cdots\left(n_{0} n_{0}+2\right)$. To any permutation $t$ in $S_{n}$ we associate the $n$ by $n$ permutation matrix defined by $M(t)=\left(\delta_{i t, j}\right)_{1 \leq i, j \leq n}$. Let $\varepsilon$ be the row vector of size $n$ all of whose entries are 1 . Define the two $2 n+1$ by $2 n+1$ matrices $A$ and $B$ by

$$
A=\left(\begin{array}{ccc}
M(a) & 0 & 0 \\
0 & M(a) & 0 \\
0 & 0 & 1
\end{array}\right), \quad B=\left(\begin{array}{ccc}
M(b) & 0 & -\varepsilon^{t} \\
0 & M(a b) & -\varepsilon^{t} \\
0 & 0 & -1
\end{array}\right)
$$

Then we have

$$
R_{1}(a)=\left(\begin{array}{cc}
A & 0 \\
0 & A
\end{array}\right) \quad \text { and } \quad R_{1}(b)=\left(\begin{array}{cc}
B & 0 \\
0 & B^{t}
\end{array}\right)
$$


Suppose $\pi_{2}: S \rightarrow S / D_{2 n}$ is the quotient map for the action of $D_{2 n}$ on $S$ determined by $\psi_{2}$. We repeat the same constructions as before. Define $x_{1}, \ldots, x_{2 n+1}$ as above for $\psi_{1}$ and

$$
\begin{aligned}
y_{1} & =Y_{1}\left(b a^{n_{0}}\right) X_{2}(b) \cdots X_{2}\left(b a^{n_{0}-1}\right)-Y_{2}\left(a^{n_{0}}\right)+x_{n_{0}+1}+\cdots+x_{n}, \\
y_{k} & =a^{k-1} \cdot y_{1}, \quad 2 \leq k \leq n, \\
y_{n+1} & =-Y_{2}(1), \\
y_{n+k} & =a^{k-1} \cdot y_{n+1}, \quad 2 \leq k \leq n, \\
y_{2 n+1} & =Y_{2}(b a)-Y_{2}(1)+x_{1}+\cdots+x_{n_{0}}+x_{2 n+1} .
\end{aligned}
$$

Again $\left\{x_{1}, \ldots, y_{2 n+1}\right\}$ is a canonical basis of $H_{1}(S, \mathbb{Z})$. By construction $R_{2}(a)=R_{1}(a)$. Also, the action of $b$ on $x_{1}, \ldots, x_{2 n+1}$ is the same as for $\psi_{1}$. An easy but tedious calculation yields

$$
R_{2}(b)=\left(\begin{array}{cc}
B & -B D \\
0 & B^{t}
\end{array}\right)
$$

where

$$
D=\left(\begin{array}{ccc}
0 & D_{0} & 0 \\
D_{0}^{t} & \varepsilon^{t} \varepsilon & \varepsilon^{t} \\
0 & \varepsilon & 0
\end{array}\right)
$$

and

$$
D_{0}=I+M(a)+\cdots+M(a)^{n_{0}-1} .
$$

Note that $\varepsilon^{t} \varepsilon=M(a) D_{0}+D_{0}^{t}=I+M(a)+\cdots+M(a)^{n-1}, \quad D=D^{t}$, and $-B D=D B^{t}$.

We now want to show that, even after composing with an automorphism of $D_{2 n}, R_{1}$ cannot be conjugated to $R_{2}$ in $\mathrm{Sp}(4 n+2, \mathbb{Z})$. In fact we can show that $R_{1}\left(D_{2 n}\right)$ cannot be conjugated to $R_{2}\left(D_{2 n}\right)$ in $\operatorname{SL}(4 n+2, \mathbb{Z})$. Consider the induced actions of $D_{2 n}$ on $H_{1}(S, \mathbb{R}) / H_{1}(S, \mathbb{Z})$, the real form of the Jacobi variety $J(S)$ of $S$. Let $\nu \in \mathbb{R}^{4 n+2}$. Then $R_{1}(a) \nu=R_{2}(a) \nu \equiv \nu \bmod \mathbb{Z}$ if and only if $\nu \equiv\left(r_{1} \varepsilon, r_{2} \varepsilon, r_{3}, s_{1} \varepsilon, s_{2} \varepsilon, s_{3}\right)^{t} \bmod \mathbb{Z}$, for some $r_{1}, r_{2}, r_{3}, s_{1}, s_{2}, s_{3} \in$ $\mathbb{R}$. Assuming $\nu$ has this form we see that

$$
R_{1}(b) \nu \equiv \nu \bmod \mathbb{Z} \quad \text { iff } \quad r_{3} \equiv n s_{1}+n s_{2}+2 s_{3} \equiv 0 \bmod \mathbb{Z},
$$

while

$$
R_{2}(b) \nu \equiv \nu \bmod \mathbb{Z} \quad \text { iff } \quad r_{3}-n_{0} s_{2} \equiv n_{0} s_{1}+n_{0} s_{2}+s_{3} \equiv 0 \bmod \mathbb{Z} .
$$

In particular, if we consider elements of order 2 in $J(S)$, that is, we restrict $r_{i}$ and $s_{i}$ to be 0 or $\frac{1}{2}$, then we find that $R_{1}\left(D_{2 n}\right)$ fixes $\left(\mathbb{Z}_{2}\right)^{5}$, while $R_{2}\left(D_{2 n}\right)$ fixes $\left(\mathbb{Z}_{2}\right)^{4}$. Thus $R_{1}\left(D_{2 n}\right)$ cannot be conjugate to $R_{2}\left(D_{2 n}\right)$ in $\operatorname{SL}(4 n+2, \mathbb{Z})$.

Remark. Suppose the matrix $E \in \operatorname{Sp}(4 n+2, \mathbb{Q})$ has the form

$$
E=\left(\begin{array}{cc}
I & E_{0} \\
0 & I
\end{array}\right)
$$

where $E_{0}^{t}=E_{0}$. Then for all $c$ in $D_{2 n}, E^{-1} R_{1}(c) E=R_{2}(c)$ if and only if

$$
A E_{0}-E_{0} A=0 \text { and } B E_{0}-E_{0} B^{t}=-B D \text {. }
$$

We have a solution to these equations readily available, that is, $E_{0}=-\frac{1}{2} D$. Thus $R_{1}\left(D_{2 n}\right)$ is conjugate to $R_{2}\left(D_{2 n}\right)$ in $\operatorname{Sp}(4 n+2, \mathbb{Q})$. 
This also shows that 2 is the only "bad" prime. Suppose $m=2 k+1$ is any odd positive integer. Taking $E_{0}=k D$ and reducing modulo $m$ we see that $R_{1}\left(D_{2 n}\right)$ is conjugate to $R_{2}\left(D_{2 n}\right)$ in $\operatorname{Sp}\left(4 n+2, \mathbb{Z}_{m}\right)$. This example is related to Theorem 4 in [3].

Remark. This example should be compared to those in [5]. There Gilman and Patterson examine the action of $\mathbb{Z}_{p}<\operatorname{Aut}(S)$, for $p$ prime, first on a noncanonical basis of $H_{1}(S, \mathbb{Z})$. The result depends only on $g, p$, and the number of fixed points. It is only when finding the action on a canonical basis that the rotation numbers play a role. Thus actions of $\mathbb{Z}_{p}$ having the same number of fixed points yield representations conjugate in $\operatorname{SL}(2 g, \mathbb{Z})$, but not conjugate in $\mathrm{Sp}(2 g, \mathbb{Z})$.

\section{REFERENCES}

1. I. Bernstein and A. Edmonds, On the classification of generic branched coverings of surfaces, Illinois J. Math. 28 (1984), 64-82.

2. S. A. Broughton, The homology and higher representations of the automorphism group of a Riemann surface, Trans. Amer. Math. Soc. 300 (1987), 153-158.

3. C. J. Earle, On the moduli of closed Riemann surfaces with symmetries, Advances in the Theory of Riemann Surfaces, Ann. of Math. Stud., no. 66, Princeton Univ. Press, Princeton, N.J., pp. 119-130.

4. H. Farkas and I. Kra, Riemann surfaces, Graduate Texts in Math., vol. 71, Springer-Verlag, Berlin and New York, 1980.

5. J. Gilman and D. Patterson, Intersection matrices for bases adapted to automorphisms of a compact Riemann surface, Riemann Surfaces and Related Topics, Ann. of Math. Stud., no. 97, Princeton Univ. Press, Princeton, N.J., 1981, pp. 149-166.

6. I. Guerrero, Holomorphic families of compact Riemann surfaces with automorphisms, Illinois J. Math. 26 (1982), 212-225.

7. W. J. Harvey, On branch loci in Teichmüller space, Trans. Amer. Math. Soc. 153 (1971), 387-399.

8. W. J. Harvey and C. Machlachlan, On mapping class groups and Teichmüller spaces, Proc. London Math. Soc. 30 (1975), 496-512.

9. S. Kerckhoff, The Nielsen realization problem, Ann. of Math. (2) 117 (1983), 235-265.

10. A. Kuribayashi, On analytic families of compact Riemann surfaces with non-trivial automorphism, Nagoya Math. J. 28 (1966), 119-165.

11. I. Kuribayashi, On automorphism groups of a curve as linear groups, J. Math. Soc. Japan 39 (1987), 51-77.

12. A. M. Macbeath and D. Singerman, Spaces of subgroups and Teichmüller space, Proc. London Math. Soc. 31 (1975), 211-256.

13. W. Magnus, A. Karass, and D. Solitar, Combinatorial group theory, Dover, New York, 1976.

14. H. Popp, Stratifikation von Quotientenmannigfaltigkeiten (in Characteristik 0) und insbesondere der Modulmannigfaltigkeiten für Kurven, J. Reine Angew. Math. 250 (1971), 12-41.

15. J. F. X. Ries, The Prym variety for a cyclic unramified cover of a hyperelliptic surface, J. Reine Angew. Math. 340 (1983), 59-69.

16. __ The splitting of some Jacobi varieties using their automorphism groups, preprint.

17. D. Singerman, Subgroups of Fuchsian groups and finite permutation groups, Bull. London Math. Soc. 2 (1970), 319-323.

18. __ Finitely maximal Fuchsian groups, J. London Math. Soc. 6 (1972), 29-38.

Department of Mathematical Sciences, State University of New York at Binghamton, Binghamton, New York 13902

Current address: Department of Mathematics, East Carolina University, Greenville, North Carolina 27858-4353 